\title{
Bremsstrahlung cannon design for Shock Ignition relevant regime
}

P. Koester, ${ }^{a}$ F. Baffigi, G. Cristoforetti, L. Labate, and L.A. Gizzi ${ }^{\text {b) }}$

Intense Laser Irradiation Laboratory, Istituto Nazionale di Ottica, Consiglio Nazionale delle Ricerche, Via G. Moruzzi 1, 56124 Pisa, Italy

S. Baton and M. Koenig

Laboratoire pour l'Utilisation des Lasers Intenses, LULI, CNRS-Ecole

A. Colaïtis, D. Batani, A. Casner, D. Raffestin, A. Tentori, and J. Trela

Centre Lasers Intenses et Applications, CELIA, Université de Bordeaux-CNRS-CEA, UMR 5107, F-33405 Talence, France

C. Rousseaux, G. Boutoux, S. Brygoo, L. Jacquet, and C. Reverdin

CEA, DAM, DIF, F-91297 Arpajon, France

E. Le Belc) and L. Le-Deroff

CEA, DAM, CESTA, F-33114 Le Barp, France

W. Theobald

Laboratory for Laser Energetics, University of Rochester, Rochester,

New York 14623, USA

K. Shigemori

Institute of Laser Engineering, University of Osaka 565-0871,

Japan 
We report on the optimization of a Bremsstrahlung Cannon (BSC) design for the investigation of laser-driven fast electron populations in a Shock-Ignition relevant experimental campaign at the LMJ-PETAL facility. In this regime with laser intensities of $10^{15}-10^{16} \mathrm{~W} / \mathrm{cm}^{2}$, fast electrons with energies $\leq 100 \mathrm{keV}$ are expected to be generated through Stimulated Raman Scattering (SRS) and Two Plasmon Decay (TPD) instabilities. The main purpose of the BSC in our experiment is to identify the contribution to X-ray emission from Bremsstrahlung of fast electrons originating from SRS and TPD, with expected temperatures of $40 \mathrm{keV}$ and $95 \mathrm{keV}$, respectively. Data analysis and reconstruction of the distributions of X-ray photons incident on the bremsstrahlung cannon are described.

a) corresponding author:petra.koester@ino.cnr.it

b) corresponding author:leonidaantonio.gizzi@ino.cnr.it

c) Also at Centre Lasers Intenses et Applications, CELIA, Université de Bordeaux-CNRS-CEA, UMR 5107, F-33405 Talence, France 


\section{INTRODUCTION}

In the Shock Ignition (SI) approach to Inertial Confinement Fusion (ICF) the compression phase and ignition phase are separated ${ }^{1}$. In the first phase, symmetric laser irradiation compresses the outer shell of the fuel capsule, generating a large-scale plasma corona. After the compression, a laser intensity spike is envisaged to drive a strong shock $(\sim 300$ Mbar $)$ in the precompressed fuel capsule to generate ignition conditions. In order to produce such a strong shock, the laser intensity must reach values of $10^{15}-10^{16} \mathrm{~W} / \mathrm{cm}^{2}$. It is wellknown ${ }^{2}$, that in this interaction regime parametric instabilities such as Stimulated Raman Scattering $(\mathrm{SRS})^{3-6}$, Two Plasmon Decay $(\mathrm{TPD})^{5,7}$ and Stimulated Brillouin Scattering $(\mathrm{SBS})^{8,9}$ are driven in the long scalelength plasma generated during the compression phase. These instabilities result in reflected laser light (SRS, SBS) and the generation of nonthermal electron populations $(\mathrm{SRS}, \mathrm{TPD})^{10}$, the so-called fast electrons. One of the major open issues within the SI approach is the effect of the fast electrons on the ability to drive a strong shock ${ }^{11}$. On the one hand, the fast electrons might have the detrimental effect of preheating the precompressed fuel, resulting in less efficient shock formation. On the other hand, low-energy fast electrons $(<100 \mathrm{keV})$ can be stopped inside the high-density precompressed shell enhancing the shock formation ${ }^{12}$. Measuring the fast electron properties (conversion efficiency, temperature) simultaneously with the shock pressure is therefore of a great importance in the SI relevant regime.

Fast electron properties have been studied through direct measurement of the escaping component of fast electrons with magnetic dipole spectrometers ${ }^{13}$ or via dosimetric techniques $^{14}$, and indirect measurements such as bremsstrahlung X-ray measurements ${ }^{15,16}$ and measurements of $K_{\alpha}$ emission $^{17,18}$, also from buried fluorescent layers at different depth inside the target $^{19}$. In the experimental conditions considered here the target is thick with respect to the CSDA (Continuous Slowing Down Approximation) stopping range of the electron energies. Thus the direct measurement of the fast electron energies with a magnetic dipole spectrometer is not suitable, as only electrons exiting the target can be detected. The other two diagnostic techniques have been used in the experiment carried out at the Laser Megajoule-PETawatt Aquitaine Laser (LMJ-PETAL) facility. Here we focus on the bremsstrahlung measurements. The discussion of the results from fluorescence emission can be found elsewhere ${ }^{20}$. 
BSCs have been widely used in relativistic laser-matter interaction studies with thin targets $^{21-24}$. In that case, the CSDA range of the major part of the fast electrons largely exceeds the target thickness, mainly because of the much higher fast electron temperatures. The fast electron energy loss inside the target can be neglected and the integration of the bremsstrahlung cross-section over the fast electron distribution (usually assumed to be Maxwellian) yields a good approximation ${ }^{15}$ of the bremsstrahlung emission produced. In the experimental conditions considered here, most of the fast electrons are stopped inside the target of mm-thickness. Electron energy losses, secondary particle generation and bremsstrahlung reabsorption inside the target need to be taken into account. Thus, Monte Carlo simulations are used to link the fast electron population to its bremsstrahlung spectrum for the calculation of the expected photon distribution emitted from the target.

Here we concentrate on the first phase of the data analysis, i.e. the reconstruction of the X-ray photon spectrum entering the BSC. The retrieval of the fast electron distribution from the photon spectrum will be the subject of future work. The analysis procedure for the retrieval of the photon distribution follows those reported in previous studies ${ }^{22,25}$. Similarly to those works, a response function base is constructed. Whereas the response functions in $^{22,25}$ are constructed for incoming photon energy bins, here a base of Maxwell-Boltzmann photon distributions in a range of temperatures is used. It was shown that the fast electrons produced through parametric instabilities can be well approximated with multi-temperature Maxwellian distributions ${ }^{26}$. Therefore, the bremsstrahlung photon distribution originating from the interaction of the fast electrons with the target material is expected to be well approximated by a multi-temperature Maxwell-Boltzmann distribution. $\operatorname{In}^{22}$ the response function is calculated using 1-dimensional Monte Carlo simulations, whereas in ${ }^{25}$ the cumulative transmission of the filters in the BSC stack is calculated for a large parameter space. In the study reported here, the BSC response is calculated using 3-dimensional Monte Carlo simulations, which take into account secondary particle emission and reabsorption inside the BSC stack, its housing and its shielding.

The paper is structured in the following way: In section II we describe the optimization of the BSC for the detection of the expected Bremsstrahlung spectrum, in section III we explain the analysis procedure and in section IV we reports the first experimental results obtained with the BSC design. In the last section V, conclusions are drawn. 


\section{BREMSSTRAHLUNG CANNON DESIGN FOR SI RELEVANT REGIME}

In the typical BSC design, a stack of Image Plate (IP) layers, separated by appropriately selected filters is used to detect X-ray photons ${ }^{21-23,25}$. The stack is enclosed in a container and shielded against scattered radiation, as well as electrons escaping the target. After exposure, IPs are scanned to retrieve the deposited dose, layer by layer. The selection of the filter materials and thicknesses is optimized for the expected photon distribution, as described in the following.

In our experiment, the expected peak laser intensity is $7 \times 10^{15} \mathrm{~W} / \mathrm{cm}^{2}$. In this interaction regime, the electron distribution function expected from simulations can be well approximated by a three-temperature Maxwellian distribution. In particular, fast electrons with a temperature $T$ given by $k_{B} T=45 \mathrm{keV}$ are expected to be generated through SRS with a conversion efficiency from laser energy to electron kinetic energy of 6-10\%, the second component $\left(k_{B} T=95 \mathrm{keV}\right)$ is due to TPD with an expected conversion efficiency of 1.5$2.5 \%$ and the third component originates from thermal plasma electrons with an expected temperature of $k_{B} T$ of $4-5 \mathrm{keV}$. The main objective guiding the design of the BSC stack is the possibility to distinguish between the two contributions originating from SRS and TPD and to measure their respective fast electron temperatures and conversion efficiencies.

In order to optimize the bremsstrahlung cannon stack of filters and IPs, the expected signal on each IP layer is estimated through Monte Carlo simulations with GEANT4 ${ }^{27}$ using Penelope low energy electromagnetic physics model ${ }^{28}$, which best reproduces bremsstrahlung emission from low-energy $(<3 \mathrm{MeV})$ electrons ${ }^{29}$. The simulations are set up using the detailed geometry of the target and its holder as well as the BSC and its cassette holder. In these simulations the contribution originating from thermal plasma electrons is neglected, as the low energy bremsstrahlung photons will deposit their energy in the very first layers of the stack only. A two-step procedure was followed: in the first step, Maxwellian fast electron distributions with the expected temperatures due to SRS and TPD instabilities are separately taken as input to the GEANT4 simulations and the resulting X-ray photon distribution (predominantly bremsstrahlung emission) at the entrance of the BSC stack is retrieved.

In the second step, the resulting photon distributions are parametrized and used sepa- 
TABLE I. Filter materials and thicknesses before each IP layer of the BSC stack configuration.

\begin{tabular}{|c|l|l||c|l|l||c|l|l|}
\hline $\begin{array}{c}\text { Filter } \\
\text { no. }\end{array}$ & Material & $\begin{array}{l}\text { Thickness } \\
{[\mathrm{mm}]}\end{array}$ & $\begin{array}{c}\text { Filter } \\
\text { no. }\end{array}$ & Material & $\begin{array}{l}\text { Thickness } \\
{[\mathrm{mm}]}\end{array}$ & $\begin{array}{l}\text { Filter } \\
\text { no. }\end{array}$ & Material & $\begin{array}{l}\text { Thickness } \\
{[\mathrm{mm}]}\end{array}$ \\
\hline 1 & $\mathrm{Al} / \mathrm{Mo}$ & $2.0 / 2.0$ & 10 & $\mathrm{Ta}$ & 0.100 & 19 & $\mathrm{~Pb}$ & 1.0 \\
2 & $\mathrm{Al}$ & 0.090 & 11 & $\mathrm{Ta}$ & 0.100 & 20 & $\mathrm{~Pb}$ & 1.0 \\
3 & $\mathrm{Ti}$ & 0.125 & 12 & $\mathrm{Au}$ & 0.250 & 21 & $\mathrm{~Pb}$ & 1.0 \\
4 & $\mathrm{Fe}$ & 0.125 & 13 & $\mathrm{Au}$ & 0.250 & 22 & $\mathrm{~Pb}$ & 1.0 \\
5 & $\mathrm{Cu}$ & 0.100 & 14 & $\mathrm{~Pb}$ & 0.250 & 23 & $\mathrm{~Pb}$ & 1.0 \\
6 & $\mathrm{Mo}$ & 0.100 & 15 & $\mathrm{~Pb}$ & 0.250 & 24 & $\mathrm{~Pb}$ & 1.0 \\
7 & $\mathrm{Ag}$ & 0.150 & 16 & $\mathrm{~Pb}$ & 0.500 & 25 & $\mathrm{~Pb}$ & 6.0 \\
8 & $\mathrm{Ag}$ & 0.300 & 17 & $\mathrm{~Pb}$ & 0.500 & & & \\
9 & $\mathrm{Ag}$ & 0.300 & 18 & $\mathrm{~Pb}$ & 1.0 & & & \\
\hline
\end{tabular}

rately as input to GEANT4 simulations of the BSC stack, and the deposited energy per simulated photon on each IP active layer is measured in the simulations. The obtained results are than multiplied by the expected photon numbers and converted to PSL/pixel for a pixel size of $100 \times 100 \mu \mathrm{m}^{2}$. Simulations are run for various configurations of filter materials and thicknesses, in order to optimize the stack configuration for enhanced differences between the signal originating from SRS and TPD bremsstrahlung photons. The filters are optimized to ensure sufficient sampling of the high energy region of the photon distribution, where the TPD component with expected temperature of $95 \mathrm{keV}$ dominates the signal in the IP active layers.

The optimized stack comprises 25 disk-shaped IPs and filters with increasing attenuation between the IPs, as shown in table I. This relatively large number of IP layers was chosen in order to allow the retrieval of a three-temperature distribution from the experimental data. To widen the dynamic range of our BSC, the stack was split in two channels by placing two different half circle filters in front of the optimized stack, one consisting of $2 \mathrm{~mm}$ of $\mathrm{Al}(\mathrm{Al}$ filter channel) and the other of $2 \mathrm{~mm}$ of Mo (Mo filter channel) as shown in Figure 1.

In the graphs of Figures 2 and 3 the resulting signal on the different IP layers for the expected incoming photon distributions (Figure 4) is shown. As shown in the graphs, the low energy component is strongly attenuated in the plot of Figure 3, due to the presence of 


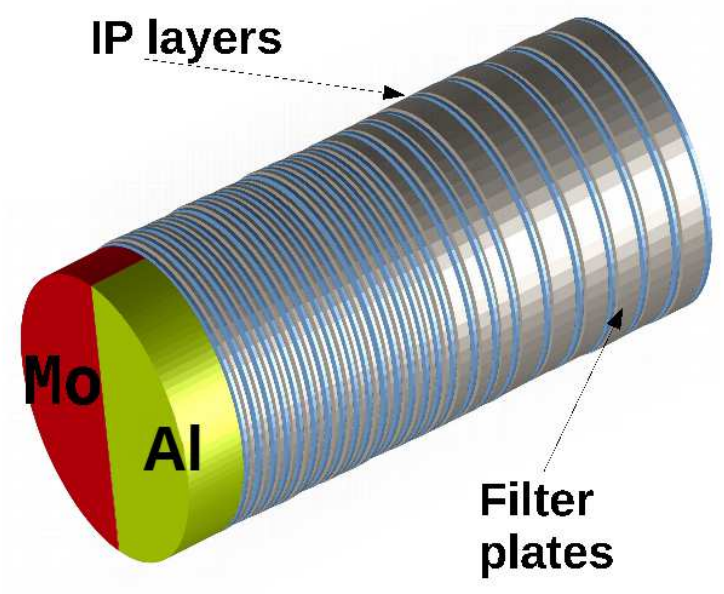

FIG. 1. Schematic view of the Bremsstrahlung cannon stack with split front filter.

the Mo filter. Most importantly, in both channels the signal on the IP layers up to layer 15 is mainly due to SRS, while from layer 15 onward is dominated by the TPD component, in spite of the much weaker incident TPD signal, nearly one order of magnitude less than the incident SRS signal. This behaviour in the SRS and TPD signal vs. IP layer is key to the identification of their respective contributions.

\section{DATA ANALYSIS PROCEDURE}

\section{A. Data Extraction}

The IPs (Fujifilm BAS-MS) are scanned after exposure with a GE Typhoon 7000 flatbed IP scanner. The IP scanner stores the scanned values in square-root encoded 16-bit files. The PSL/pixel values are then obtained from the scans by applying the formula ${ }^{30}$ :

$$
P S L=\left(\frac{R}{100}\right)^{2}\left(\frac{P_{G E}}{2^{D}-1}\right)^{2} h(V) 10^{L / 2}
$$

where $R$ indicates the spatial resolution in $\mu \mathrm{m}, L$ is the dynamic range latitude, $P_{G E}$ is the scanned pixel value, $D$ is the bit depth of the scanned image and $h(V)$ is an empirically determined function of the photomultiplier tube voltage $V$ applied during the readout process. We observe that the first IP layers of the Al filter channel showed saturation during the first scan, so additional successive scans were necessary until no saturation was present. 


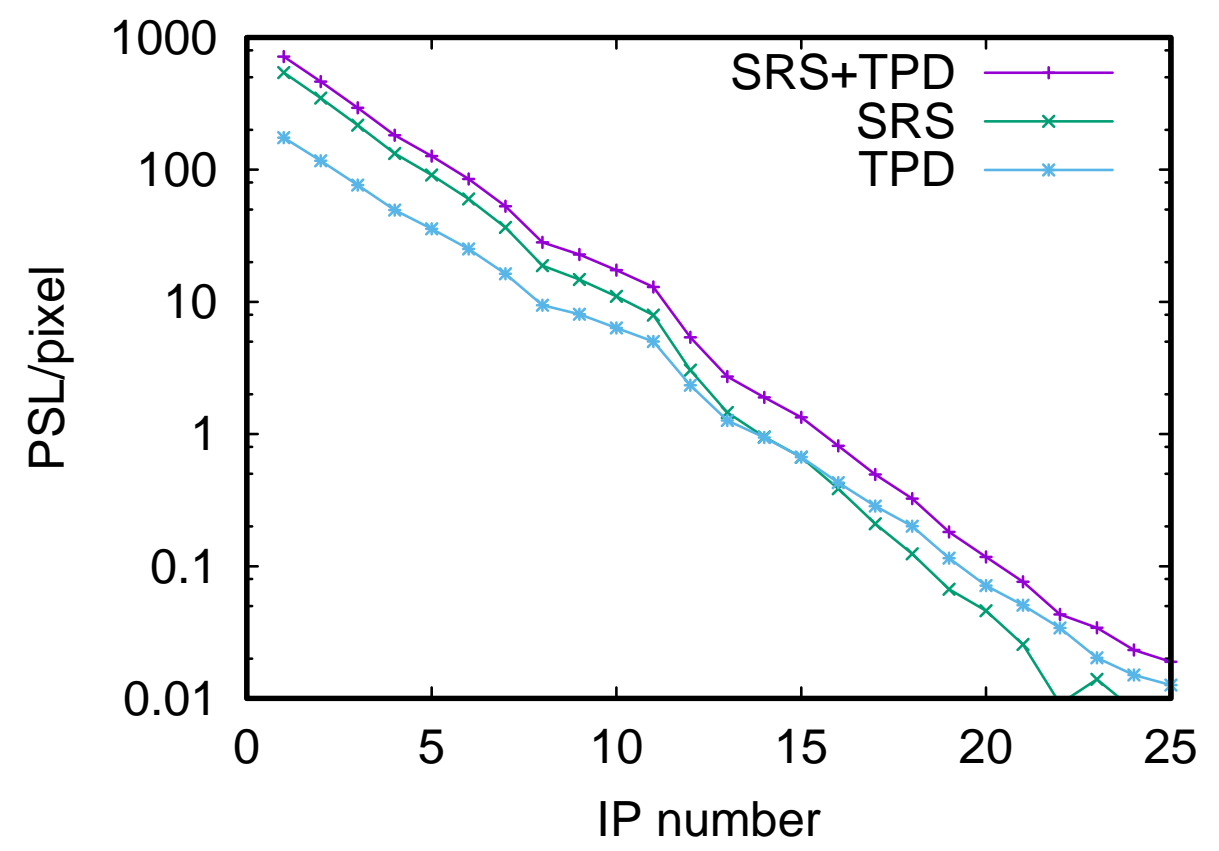

FIG. 2. Calculated response (PhotoStimulated luminescence (PSL)/pixel) of the 25 Image Plate (IP) layers in the designed Bremsstrahlung cannon stack with $2 \mathrm{~mm} \mathrm{Al} \mathrm{front} \mathrm{filter} \mathrm{(Al} \mathrm{filter} \mathrm{channel)}$ for the expected incoming bremsstrahlung photon distribution originating from Stimulated Raman Scattering (SRS) and Two Plasmon Decay (TPD) electrons.

In fact, the dynamic range of the IPs is larger than the dynamic range of the IP scanner, and each successive scan lowers the signal ${ }^{31}$, so that the full dynamic range can be recovered by successive scans.

The signal is then extracted by selecting an area in the central part of the half circle of each channel and removing the edges potentially affected by noise due to cutting imperfections or rescattering from the BSC walls. The mean and the standard deviation of the signal is calculated for the selected area for each scan. For the data set of the first shot, the exponential decrease of the signal during successive scans was verified for the various IP layers. Thus, for the IPs showing saturation in the first scan the signal is reconstructed using the ratio of the signal mean between the first and the last scan for non-saturated IP layers:

$$
P S L(k)_{\text {firstscan }}=\frac{P S L(j)_{\text {firstscan }}}{P S L(j)_{\text {lastscan }}} P S L(k)_{l a s t s c a n}
$$




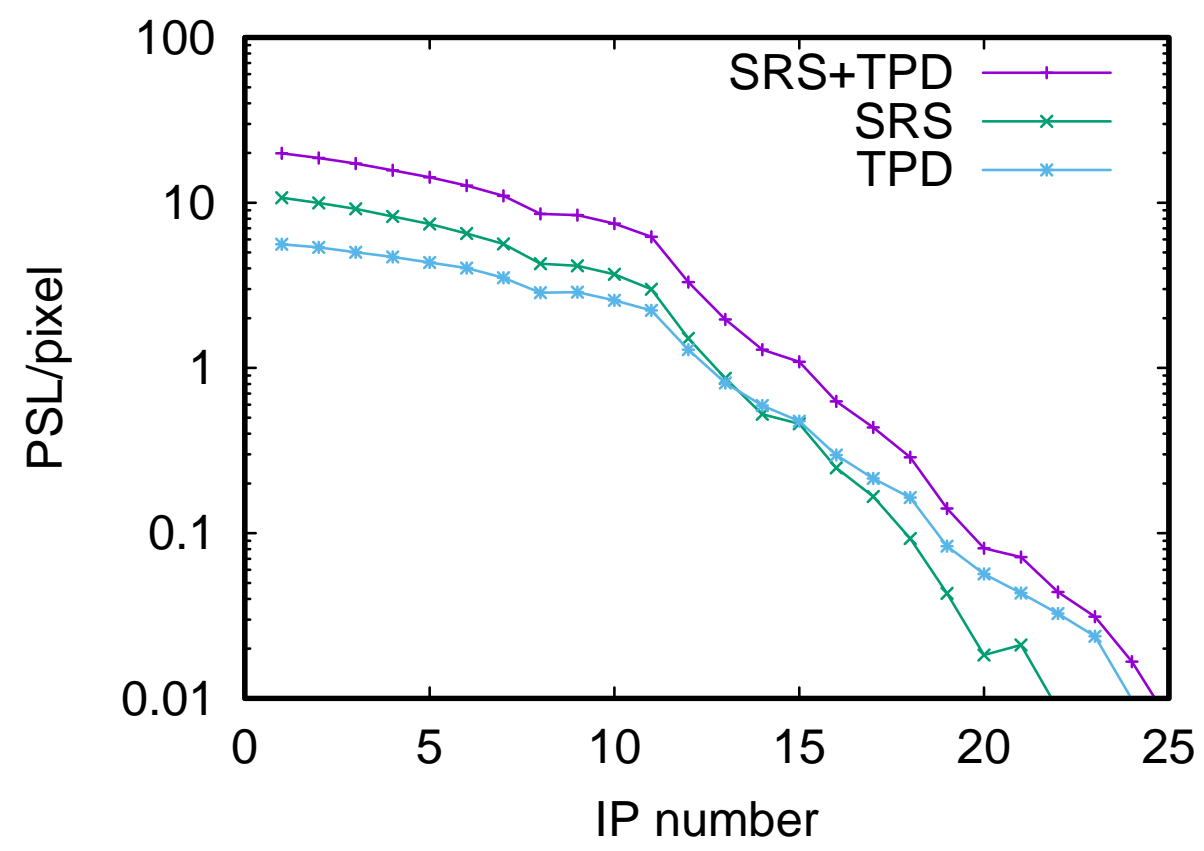

FIG. 3. Calculated response (PhotoStimulated Luminescence (PSL)/pixel) of the 25 Image Plate (IP) layers in the designed Bremsstrahlung cannon stack with $2 \mathrm{~mm}$ Mo front filter (Mo filter channel) for the expected incoming bremsstrahlung photon distribution originating from Stimulated Raman Scattering (SRS) and Two Plasmon Decay (TPD) electrons.

\section{B. Reconstruction of the bremsstrahlung photon distribution}

The photon distribution $f(\epsilon)$ impinging on the bremsstrahlung cannon stack is obtained with the assumption that it mainly consists of bremsstrahlung photons, and can be approximated with a sum of Maxwell-Boltzmann distributions with $N_{T}$ temperatures $T_{j}$ :

$$
f(\epsilon)=\sum_{j=1}^{N_{T}} \beta_{j} \frac{1}{\kappa T_{j}} \exp ^{-\frac{\epsilon}{\kappa T_{j}}},
$$

where $\epsilon$ indicates the photon energy and $\kappa$ the Boltzmann constant.

Thus, through Monte Carlo simulations a response function base for the BSC stack is built using exponential photon distributions for a discrete set of temperatures $\left\{T_{j}\right\}$ in the range between $1 \mathrm{keV}$ and $200 \mathrm{keV}$ for both the $\mathrm{Al}$ and the Mo filter channels. The energy deposition obtained per incoming photon $E_{l}^{T_{j}}$ vs. IP number $l$ is shown in the graphs in Figures 5 and 6 .

In order to find the photon distribution which best fits the measured energy deposition 


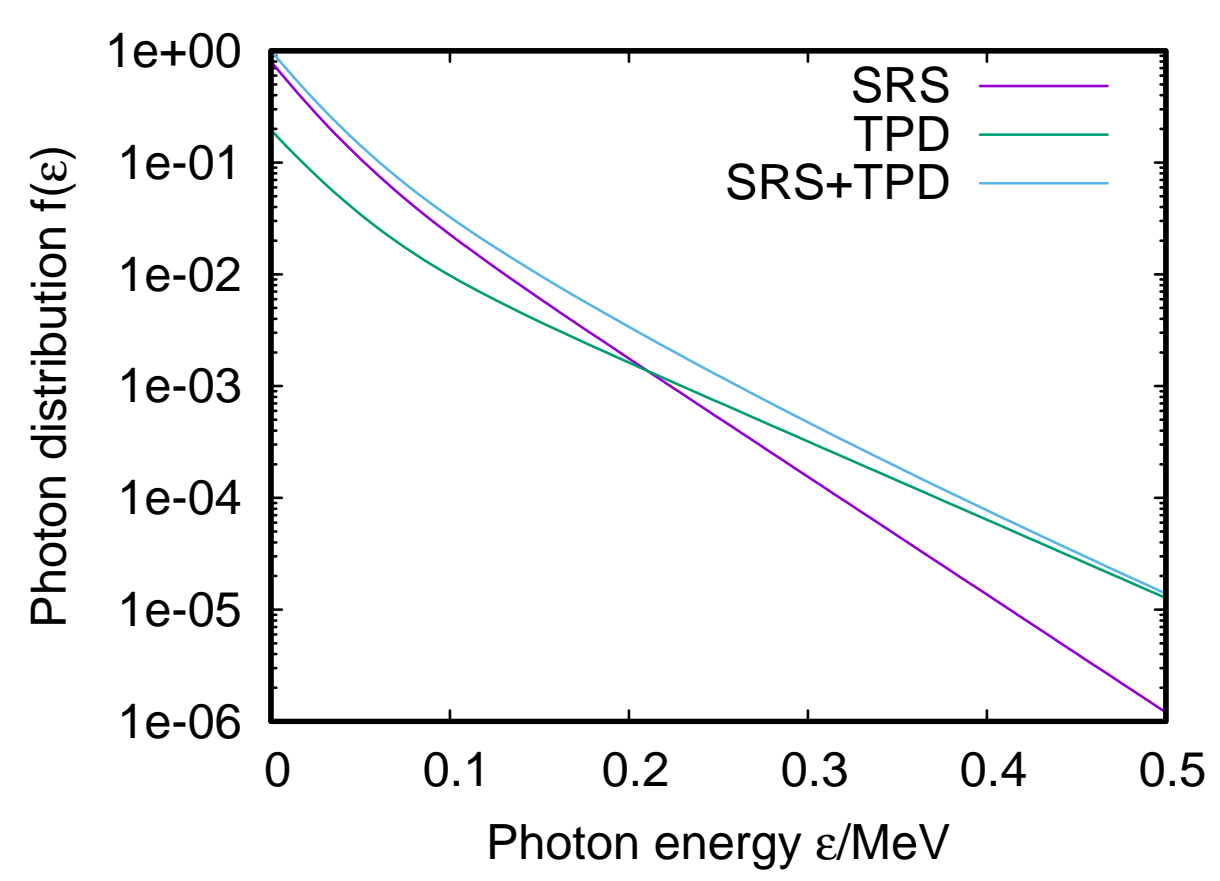

FIG. 4. The expected photon distribution function due to bremsstrahlung photons originating from Stimulated Raman Scattering (SRS) and Two Plasmon Decay (TPD) electrons.

on the different IP layers of the BSC stack, the WSSR (Weighted Sum of Squared Residuals) is evaluated for each subset of $N_{T}$ temperatures $T_{j}$ as

$$
W S S R\left(\left\{T_{j}\right\}\right)=\sum_{k=1}^{N_{I P}} \frac{\left(P S L(k) * C_{f} * C_{c a l} * N_{P}-\sum_{j=1}^{N_{T}} \beta_{j} E_{k}^{T_{j}}\right)^{2}}{\left(S t d(k) * C_{f} * C_{c a l} * N_{P}\right)^{2}},
$$

where $N_{I P}$ is the number of IPs, $P S L(k)$ is the measured PSL mean value per pixel on the $k$-th IP, $C_{f}(=1.67$ for MS-type IP) accounts for fading of the IP due to the time elapsed between exposure and readout of the $\mathrm{IP}^{32}, C_{c a l}(=1.33 \mathrm{MeV} / P S L)$ is the calibration constant relating measured PSL values to deposited energy in $\mathrm{MeV}^{33}, N_{P}$ is the number of pixels on an IP and $E_{k}^{T_{j}}$ is the energy (in $\mathrm{MeV}$ ) deposited on the $k$-th IP for an incoming photon distribution with temperature $T_{j}$ as retrieved from Monte Carlo simulations. $\beta_{j}$ are the parameters for fitting and indicate the photon number of the exponential photon distribution with temperature $T_{j}$, which best fit the experimental data. The WSSR is minimized for each subset of temperatures $\left\{T_{j}\right\}$. The minimum WSSR of each subset of temperatures is registered and the minimum value amongst all minimized WSSR is found. The corresponding subset of $N_{T}$ temperatures is the set of temperatures best fitting the experimental data. The coefficients $\beta_{j}$, which minimize the WSSR for this subset of temperatures, give the number 


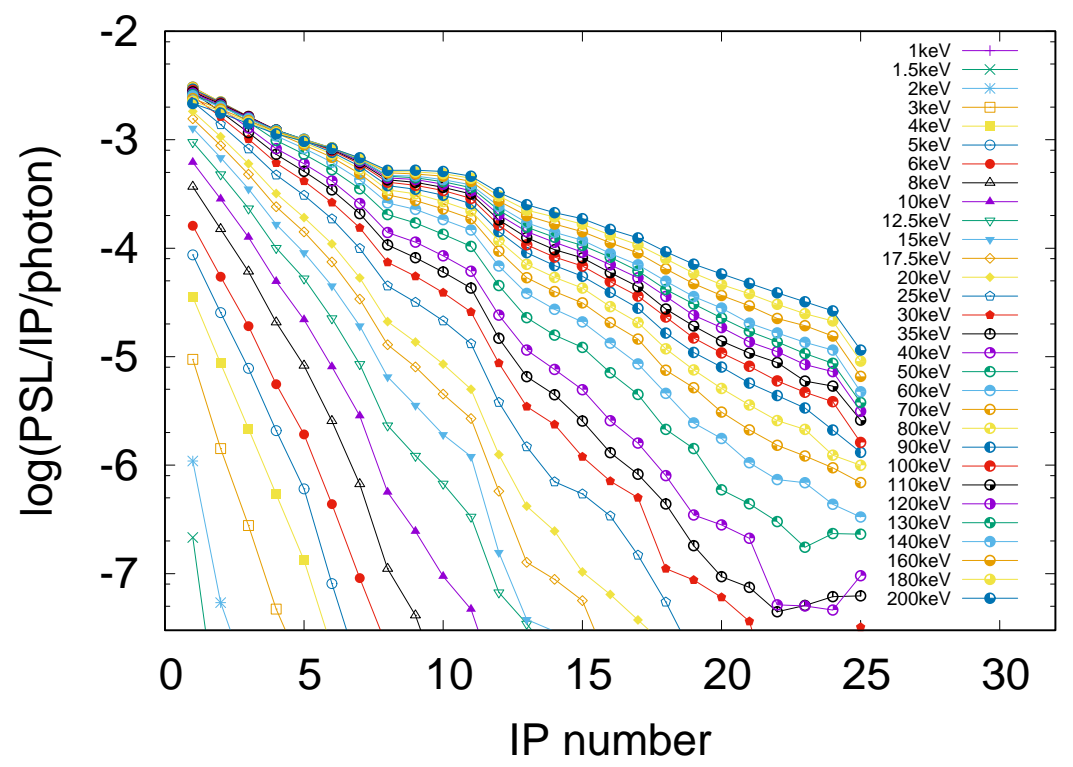

FIG. 5. Calculated response curves (PhotoStimulated Luminescence (PSL)/Image Plate (IP)/photon) of the 25 Image Plate layers in the Bremsstrahlung cannon stack to a singletemperature Maxwell-Boltzmann photon distribution for temperatures in the range $1-200 \mathrm{keV}$ for the Al filter channel.

of photons of the photon distributions with temperatures $T_{j}$. Considering the solid angle subtended by the BSC in the experimental geometry, the number of photons/sterad emitted from the target are calculated.

\section{RESULTS AND DISCUSSION}

In the experiment at LMJ-PETAL three shots were delivered with a laser intensity around $2.5 \times 10^{15} \mathrm{~W} / \mathrm{cm}^{2}$, less than the expected intensity due to non perfect beam overlapping. The first two shots were delivered on target without Smoothing by Spectral Dispersion (SSD) of the beam, whereas for the third shot the SSD was turned on. The BSC was fitted onto the end of the Cassette Radiographic Centre Chambre (CRACC) diagnostic, situated at an angle of $58.5^{\circ}$ with respect to the target normal opposite to the laser-irradiated target side. The distance to the target was $26.6 \mathrm{~cm}$ for the first two shots and $17.6 \mathrm{~cm}$ for the third shot. More details on the experimental setup and results from further diagnostics can be found elsewhere ${ }^{20}$. 


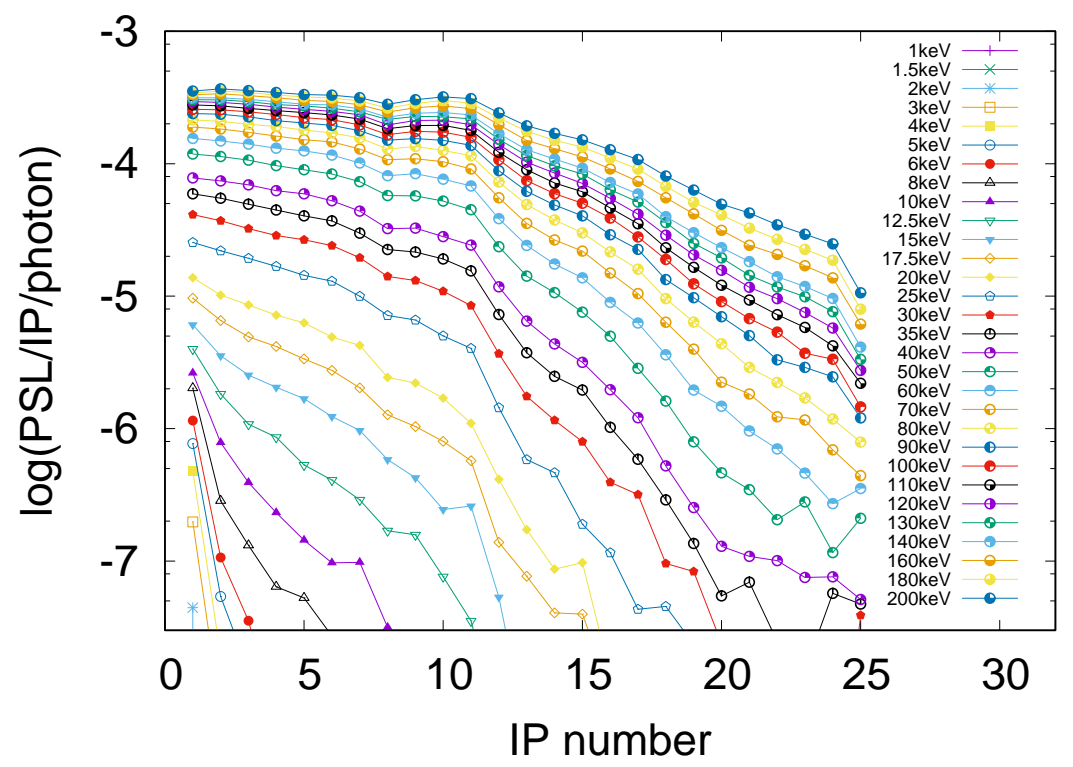

FIG. 6. Calculated response curves (PhotoStimulated luminescence (PSL)/Image Plate (IP)/photon) of the 25 Image Plate layers in the Bremsstrahlung cannon stack to a singletemperature Maxwell-Boltzmann photon distribution for temperatures in the range $1-200 \mathrm{keV}$ for the Mo filter channel.

The data points were fitted with a three-temperature X-ray photon distribution:

$$
f(\epsilon)=\sum_{i=1}^{3} \frac{N_{i}}{\kappa T_{i}} \exp ^{-\frac{\epsilon}{\kappa T_{i}}}
$$

where $N_{i}$ is the photon number per steradian in the Maxwell-Boltzmann distribution with temperature given by $\kappa T_{i}$. In the Figures 7,8 and 9 the results from the data analysis are shown for the three shots. The top panels in the figures show the minimum WSSR for each triplet of temperatures. For better visibility, only the results with WSSR 1.5 times the minimum value reached over all temperature triplets are shown. The middle panels show the maps of WSSR for all pairs $T_{2}$ and $T_{3}$ with fixed $T_{1}$ corresponding to the best fit. It should be noted that a constant WSSR along one temperature axis in these maps (as e.g. the vertical lines visible in Figure 9 (right middle panel) for Shot 3 Mo channel at temperature $T_{2}=30,35$ and $40 \mathrm{keV}$ ) indicates that the corresponding photon number vanishes, thus resulting in a 2-temperature distribution. In the bottom panels, the data measured on the IP layers together with the fit for the temperature triplet resulting in the lowest WSSR are displayed. The left panels in each figure refer to the Al filter channel and the right panels 

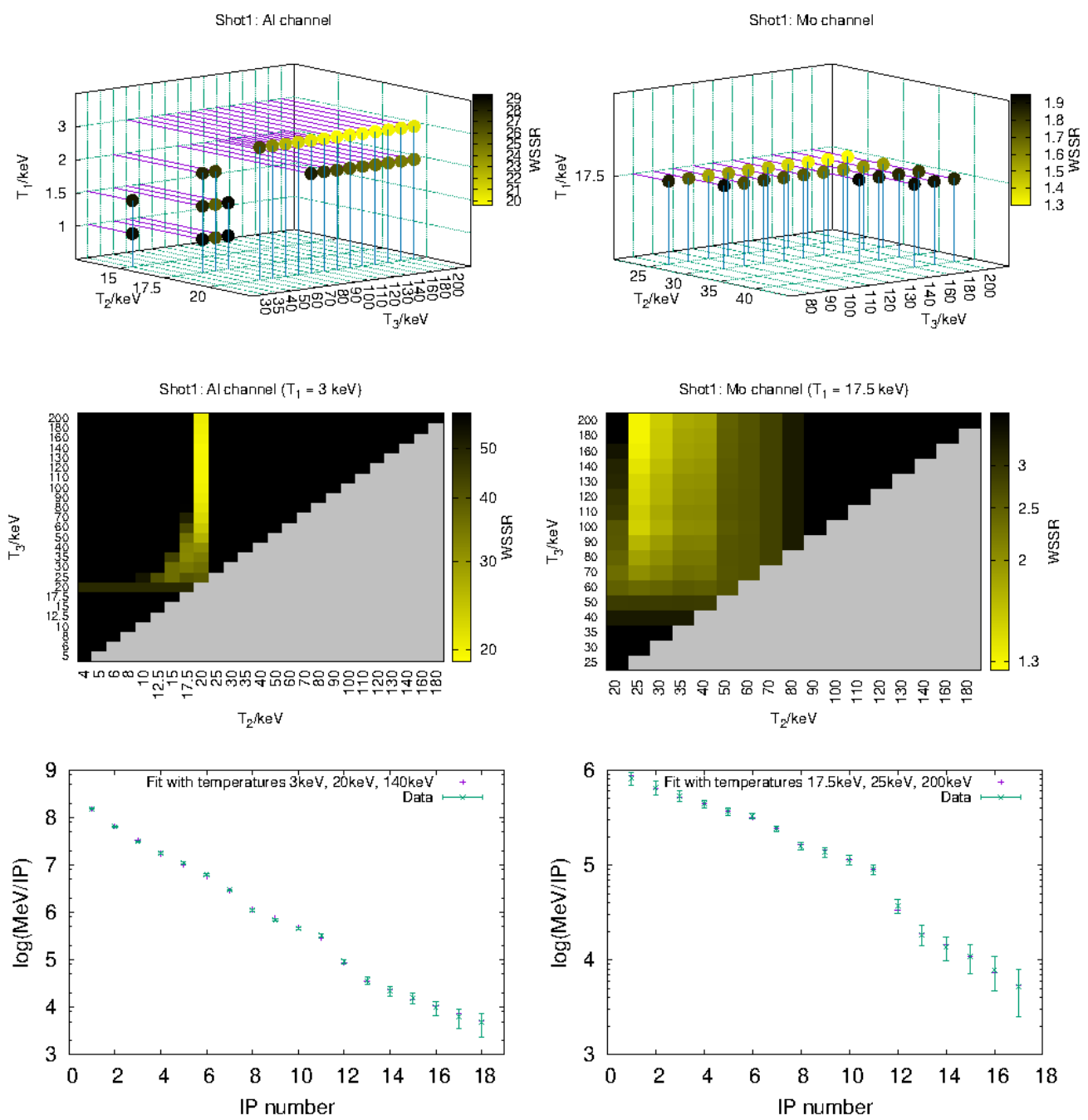

FIG. 7. Shot1: WSSR for temperature triplets $T_{1}, T_{2}$ and $T_{3}$ for the Al filter channel (top left) and the Mo filter channel (top right) and for temperature pairs $T_{2}$ and $T_{3}$ at fixed $T_{1}$ (middle panels). In the bottom panels the experimental data are shown together with the best fit (minimum WSSR) for the $\mathrm{Al}$ (left) and the Mo filter channel (right).

to the results from the Mo filter channel from the same shot. The results are summarized in Table II.

In the first shot the temperatures which best fit the experimental data are $3 \mathrm{keV}, 20 \mathrm{keV}$ and $140 \mathrm{keV}$ for the Al filter channel and $17.5 \mathrm{keV}, 25 \mathrm{keV}$ and $200 \mathrm{keV}$ for the Mo filter channel. The lowest temperatures are expected to differ for the two channels, as the low energy photons will not reach the IP layers in the case of the Mo filter due to the higher attenuation. Therefore, the intermediate temperature retrieved from the analysis of the $\mathrm{Al}$ 

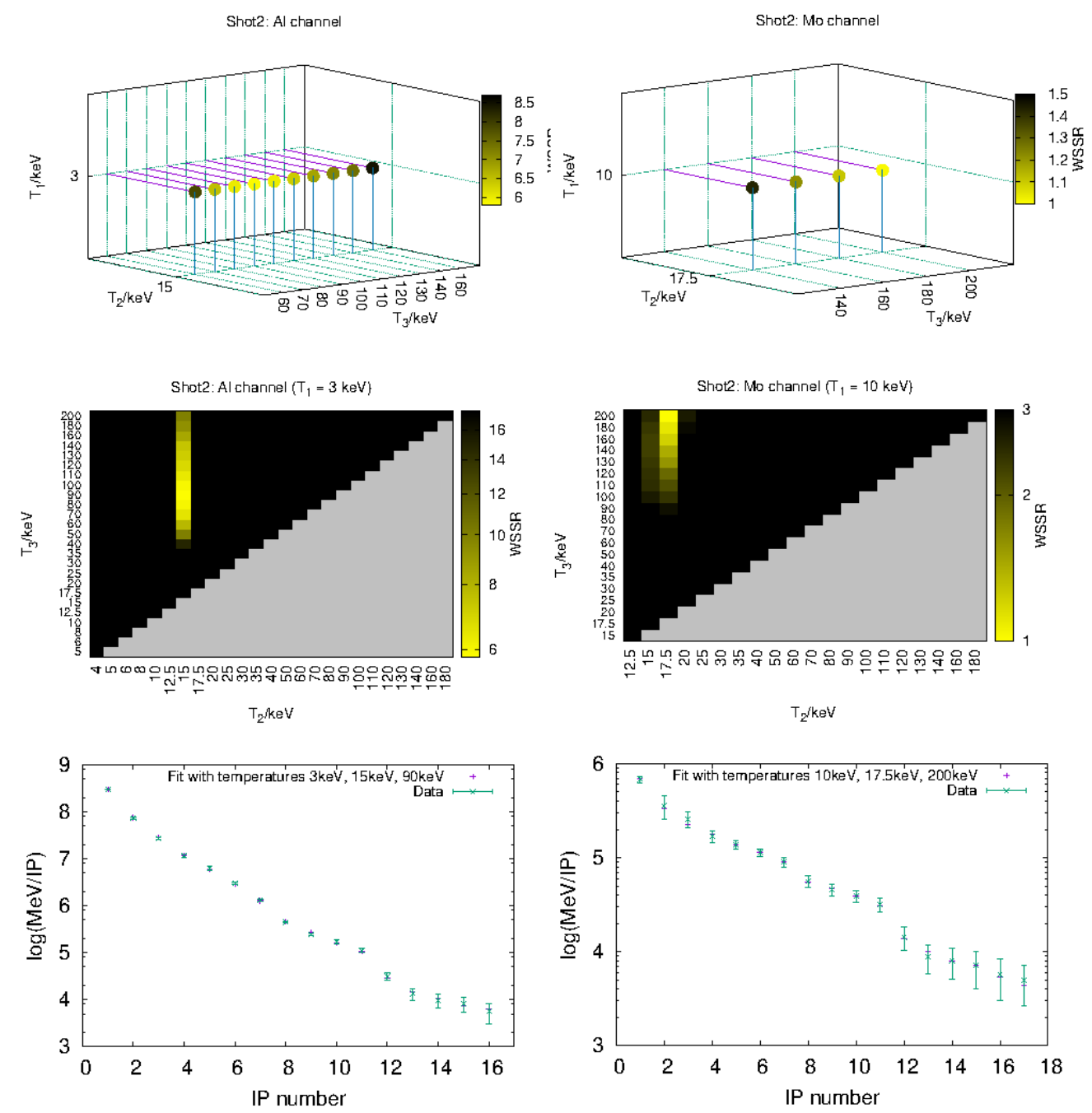

FIG. 8. Shot2: WSSR for temperature triplets $T_{1}, T_{2}$ and $T_{3}$ for the Al filter channel (top left) and the Mo filter channel (top right) and for temperature pairs $T_{2}$ and $T_{3}$ at fixed $T_{1}$ (middle panels). In the bottom panels the experimental data are shown together with the best fit (minimum WSSR) for the $\mathrm{Al}$ (left) and the Mo filter channel (right).

filter channel should be compared to the low and intermediate temperature retrieved from the analysis of the Mo filter channel data. In particular, the Al filter channel yields an intermediate temperature of $20 \mathrm{keV}$, in fairly good agreement with the combination of the retrieved temperatures $T_{1}=17.5 \mathrm{keV}$ and $T_{2}=25 \mathrm{keV}$ from the analysis of the Mo filter channel. The highest temperatures retrieved from the analysis of the two BSC channels differ significantly due to limitations of the analysis procedure. In fact, the higher temperature is affected by a large error, as can be seen in the top panels in Figure 7 . The WSSR changes 
Shot3: Al channe

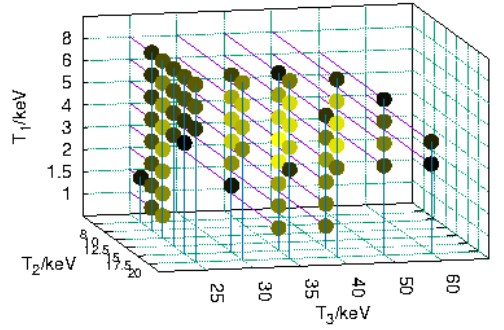

Shot3: Al channel $\left(T_{1}=4 \mathrm{keV}\right)$
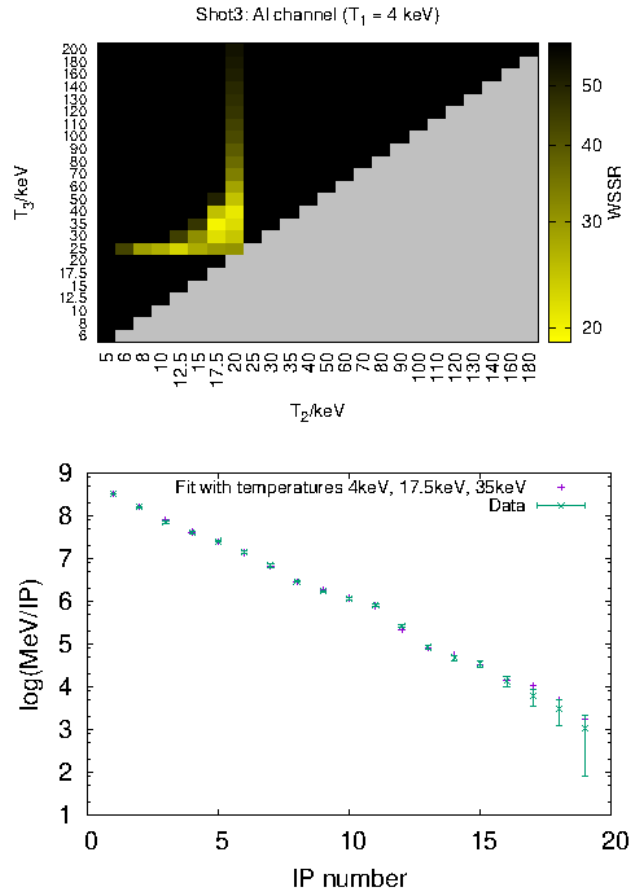

Shot3: Mo channe

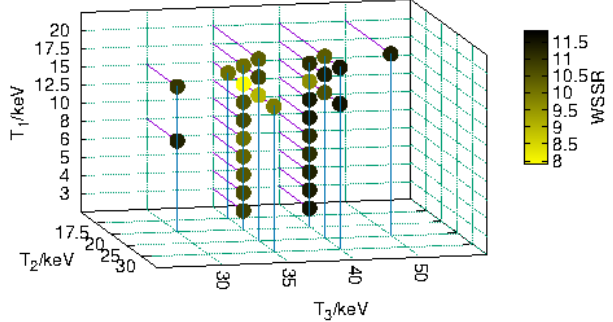

Shot3: Mo channel $\left(T_{1}=15 \mathrm{keV}\right)$
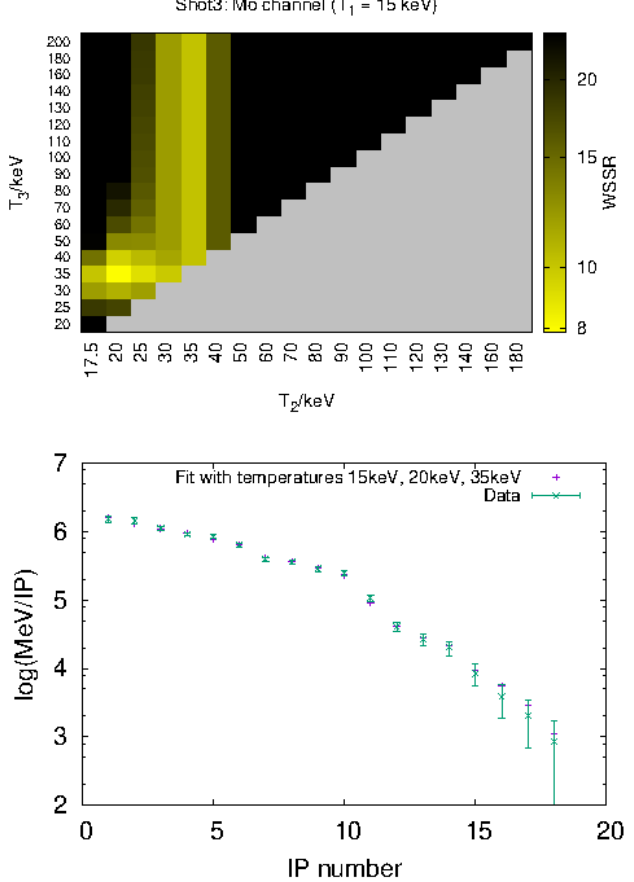

FIG. 9. Shot3: WSSR for temperature triplets $T_{1}, T_{2}$ and $T_{3}$ for the Al filter channel (top left) and the Mo filter channel (top right) and for temperature pairs $T_{2}$ and $T_{3}$ at fixed $T_{1}$ (middle panels). In the bottom panels the experimental data are shown together with the best fit (minimum WSSR) for the $\mathrm{Al}$ (left) and the Mo filter channel (right).

only slightly over a large range of the highest temperatures $T_{3}$. This is mainly due to the low photon flux on highly filtered IP layers that are more sensitive to the $T_{3}$ temperature distribution - that is, more than two orders of magnitude lower than the photon flux on the intermediate IP layers that are more sensitive to the $T_{2}$ temperature distribution (see Table II).

Very similar considerations apply to the second shot. The resulting intermediate temperatures are slightly lower than in the case of Shot $1\left(T_{2}=15 \mathrm{keV}\right.$ for the Al filter channel and 
TABLE II. Best fitting temperature triplets and photon numbers for the $\mathrm{Al}$ and the Mo filter channel for the three shots. The temperature ranges corresponding to WSSR $<1.5$ times the minimum WSSR are also given (square brackets).

\begin{tabular}{|c|c|c|c|c|c|c|}
\hline & $\begin{array}{r}T_{1} \\
{[\mathrm{keV}]}\end{array}$ & $\begin{array}{r}N_{1} \\
{\left[\frac{\text { photons }}{\text { sterad }}\right]}\end{array}$ & $\begin{array}{r}T_{2} \\
{[\mathrm{keV}]}\end{array}$ & $\begin{array}{r}N_{2} \\
{\left[\frac{\text { photons }}{\text { sterad }}\right]}\end{array}$ & $\begin{array}{r}T_{3} \\
{[\mathrm{keV}]}\end{array}$ & $\begin{array}{r}N_{3} \\
{\left[\frac{\text { photons }}{\text { sterad }}\right]}\end{array}$ \\
\hline Shot $1 \mathrm{Al}$ & $\begin{array}{r}3 \\
{[2-3]}\end{array}$ & $8.6 \times 10^{14}$ & $\begin{array}{r}20 \\
{[17.5-20]}\end{array}$ & $7.3 \times 10^{12}$ & $\begin{array}{r}140 \\
{[60-200]}\end{array}$ & $1.1 \times 10^{10}$ \\
\hline Shot $1 \mathrm{Mo}$ & $\begin{array}{r}17.5 \\
{[17.5]}\end{array}$ & $8.6 \times 10^{12}$ & $\begin{array}{r}25 \\
{[25-40]}\end{array}$ & $1.4 \times 10^{12}$ & $\begin{array}{r}200 \\
{[80-200]}\end{array}$ & $6.0 \times 10^{9}$ \\
\hline Shot $2 \mathrm{Al}$ & $\begin{array}{r}3 \\
{[3]}\end{array}$ & $3.2 \times 10^{15}$ & $\begin{array}{r}15 \\
{[15]}\end{array}$ & $8.8 \times 10^{12}$ & $\begin{array}{r}90 \\
{[60-180]}\end{array}$ & $1.8 \times 10^{10}$ \\
\hline Shot $2 \mathrm{Mo}$ & $\begin{array}{r}10 \\
{[10]}\end{array}$ & $1.9 \times 10^{13}$ & $\begin{array}{r}17.5 \\
{[17.5]}\end{array}$ & $4.5 \times 10^{12}$ & $\begin{array}{r}200 \\
{[140-200]}\end{array}$ & $5.4 \times 10^{9}$ \\
\hline Shot $3 \mathrm{Al}$ & $\begin{array}{r}4 \\
{[2-8]}\end{array}$ & $1.5 \times 10^{14}$ & $\begin{array}{r}17.5 \\
{[10-20]}\end{array}$ & $8.2 \times 10^{12}$ & $\begin{array}{r}35 \\
{[30-60]}\end{array}$ & $5.9 \times 10^{11}$ \\
\hline Shot $3 \mathrm{Mo}$ & $\begin{array}{r}15 \\
{[3-17.5]}\end{array}$ & $1.1 \times 10^{13}$ & $\begin{array}{r}20 \\
{[17.5-30]}\end{array}$ & $3.3 \times 10^{12}$ & $\begin{array}{r}35 \\
{[30-50]}\end{array}$ & $5.1 \times 10^{11}$ \\
\hline
\end{tabular}

the combination of $T_{1}=10 \mathrm{keV}$ and $T_{2}=17.5 \mathrm{keV}$ for the Mo filter channel), consistent with the slightly lower laser power (10.7 TW for Shot 1 and $9.7 \mathrm{TW}$ for Shot 2). A high temperature component is also present in Shot 2 (90 keV for Al filter channel and $200 \mathrm{keV}$ for the Mo filter channel.

The results from Shot 3 are significantly different from the first two shots. The highest temperature of the reconstructed photon distribtuion is $T_{3}=35 \mathrm{keV}$ (both for the $\mathrm{Al}$ and the Mo filter channel), significantly lower than the highest temperatures retrieved for the first two shots. In the middle right panel of Figure 9, the WSSR for $T_{3}>40 \mathrm{keV}$ is constant for the temperatures $T_{2}=30 \mathrm{keV}, 35 \mathrm{keV}$ and $40 \mathrm{keV}$. In this case the minimization procedure for the temperature triplets all yield $N_{3}=0$, resulting, in effect, two-temperature photon distributions with temperatures $T_{1}$ and $T_{2}$, and therefore the WSSR is constant. 
For this shot the temperatures $T_{2}$ and $T_{3}$ of the reconstructed photon distributions are in good agreement between the $\mathrm{Al} / \mathrm{Mo}$ filter channels. Although the photon distribution temperatures cannot be directly related to the fast electron temperatures at our experimental conditions as explained above, the absence of the high-temperature component certainly indicates that lower energy fast electrons are generated in Shot 3. This is consistent with the irradiation conditions, as the SSD smoothing technique is expected to lower the level of parametric instabilities.

\section{CONCLUSIONS}

Bremsstrahlung measurements were performed in a shock ignition relevant experiment at LMJ using a BSC detector. The BSC stack was optimized for detecting bremsstrahlung emission from fast electrons, as expected in this intensity regime, with the aim to distinguish the contributions arising from two fast electron distributions due to SRS and TPD instabilities. The first experimental results clearly show that the designed BSC stack is capable of discriminating variations of X-ray photon distributions typical of this SI regime of interaction. A three-temperature Maxwell-Boltzmann distribution, with temperatures around 2-4 $\mathrm{keV}, 15-25 \mathrm{keV}$ and $\geq 90 \mathrm{keV}$ respectively, satisfactorily fits the measured Bremsstrahlung emission. Results also clearly show that the set up of the BSC high energy component is sensitive to the change of interaction conditions of the high energy spectral component when SSD is used to reduce instabilities. The determination of the exact temperature value of the high-energy component is affected by a large uncertainty due to the low number of photons and will require further optimization that can now be carried out based on this experimental observation. Further, in order to strengthen the correlation between the X-ray spectral features and the original fast electron distributions, the detailed geometrical and physical

properties of the target should be taken into account to properly model the bremsstrahlung emission.

\section{ACKNOWLEDGMENTS}

The authors acknowledge support from the Enabling Research Project EUROFusion (Task Agreement ENR-IFE19.CEA-01, Grant agreement no. 633053) funded under Horizon2020- 
Euratom programme. The PETAL laser was designed and constructed by CEA under the financial auspices of the Conseil Regional d'Aquitaine, the French Ministry of Research, and the European Union. The CRACC diagnostic was designed and commissioned on the LMJ-PETAL facility as a result of the PETAL+ project coordinated by Université de Bordeaux and funded by the French Agence Nationale de la Recherche under grant ANR-10-EQPX-42-01 and the PetaPhys Project under grant ANR-10-IDEX-03-02. The LMJ-PETAL experiment presented in this article was supported by Association Lasers et Plasmas and by CEA. The views and opinions expressed herein do not necessarily reflect those of the European Commission.

\section{DATA AVAILABILITY}

The data that support the findings of this study are available from the corresponding author upon reasonable request.

\section{REFERENCES}

${ }^{1}$ R. Betti, C. D. Zhou, K. S. Anderson, L. J. Perkins, W. Theobald, and A. A. Solodov, "Shock ignition of thermonuclear fuel with high areal density," Physical Review Letters 98, 155001 (2007).

${ }^{2}$ W. L. Kruer, The Physics of Laser-Plasma Interactions, Frontiers in Physics, Vol. 73 (Addison-Wesley Publishing Co; Reading, MA (USA), 1988).

${ }^{3}$ W. Seka, E. A. Williams, R. S. Craxton, L. M. Goldman, R. W. Short, and K. Tanaka, "Convective stimulated raman scattering instability in uv laser plasmas," Physics of Fluids 27, 2181 (1984).

${ }^{4}$ K. Tanaka, L. M. Goldman, W. Seka, M. C. Richardson, J. M. Soures, and E. A. Williams, "Stimulated raman scattering from uv-laser-produced plasmas," Physical Review Letters 48, 1179 (1982).

${ }^{5}$ C. Rousseaux, F. Amiranoff, C. Labaune, and G. Matthieussent, "Suprathermal and relativistic electrons produced in laser-plasma interaction at $0.26,0.53$ and $1.05 \mu \mathrm{m}$ laser wavelength," Physics of Fluids B 4, 2589 (1992). 
${ }^{6}$ G. Cristoforetti, L. Antonelli, S. Atzeni, F. Baffigi, F. Barbato, D. Batani, G. Boutoux, A. Colaitis, J. Dostal, R. Dudzak, L. Juha, P. Koester, A. Marocchino, D. Mancelli, P. Nicolai, O. Renner, J. J. Santos, A. Schiavi, M. M. Skoric, M. Smid, P. Straka, and L. A. Gizzi, "Measurements of parametric instabilities at laser intensities relevant to strong shock generation," Physics of Plasmas 25, 012702 (2018).

${ }^{7}$ A. B. Langdon, B. F. Lasinski, and W. L. Kruer, "Nonlinear saturation and recurrence of the two-plasmon decay instability," Physical Review Letters 43, 1591 (1979).

${ }^{8}$ O. Willi, T. Afshar-rad, S. Coe, and A. Giulietti, "Study of instabilities in long scale-length plasmas with and without laser-beam-smooting techniques," Physics of Fluids B: Plasma Physics 2, 1318 (1990).

${ }^{9}$ T. Afshar-rad, L. A. Gizzi, M. Desselberger, F. Khattak, and O. Willi, "Evidence for whole-beam self-focusing of induced spatially incoherent laser light in large underdense plasma," Physical Review Letters 68, 942 (1992).

${ }^{10}$ G. Cristoforetti, L. Antonelli, D. Mancelli, S. Atzeni, F. Baffigi, F. Barbato, D. Batani, G. Boutoux, F. D’Amato, J. Dostal, R. Dudzak, E. Filippov, Y. J. Gu, L. Juha, O. Klimo, M. Krus, S. Malko, A. S. Martynenko, P. Nicolai, V. Ospina, S. Pikuz, O. Renner, J. J. Santos, V. T. Tikhonchuk, J. Trela, S. Viciani, L. Volpe, S. Weber, and L. A. Gizzi, "Time evolution of stimulated Raman scattering and two-plasmon decay at laser intensities relevant for shock ignition in a hot plasma," High Power Laser Science and Engineering 7, e51 (2019).

${ }^{11}$ W. Theobald, R. Nora, W. Seka, M. Lafon, K. S. Anderson, M. Hohenberger, F. J. Marshall, D. T. Michel, A. A. Solodov, C. Stoeckl, D. H. Edgell, B. Yaakobi, C. A., C. Reverdin, X. Ribeyre, A. Shvydky, A. Vallet, J. Peebles, F. N. Beg, M. S. Wei, and R. Betti, "Spherical strong-shock generation for shock-ignition inertial fusion," Physics of Plasmas 22, $056310(2015)$.

${ }^{12}$ L. Antonelli, J. Trela, F. Barbato, J. Boutoux, P. Nicolai, D. Batani, V. Tikhonchuk, D. Mancelli, A. Tentori, S. Atzeni, A. Schiavi, F. Baffigi, G. Cristoforetti, S. Viciani, L. A. Gizzi, M. Smid, O. Renner, J. Dostal, R. Dudzak, L. Juha, and M. Krus, "Laser-driven strong shocks with infrared lasers at intensity of $10^{16} \mathrm{~W} / \mathrm{cm}^{2}$," Physics of Plasmas 26, 112708 (2019).

${ }^{13}$ G. Malka and J. L. Miquel, "Experimental confirmation of ponderomotive-force electrons produced by an ultrarelativistic laser pulse on a solid target," Physical Review Letters 77, 
75 (1996).

${ }^{14}$ L. Labate, M. Galimberti, a. Giulietti, D. Giulietti, P. Köster, P. Tomassini, and L. A. Gizzi, "Study of forward accelerated fast electrons in ultrashort Ti K $\alpha$ sources," Applied Physics B 86, 229-233 (2006).

${ }^{15}$ L. Gizzi, D. Batani, V. Biancalana, A. Giulietti, and D. Giulietti, "X-ray emission from thin-foil laser-produced plasmas," Laser and Particle Beams 10, 65-74 (1992).

${ }^{16}$ M. H. Key, M. D. Cable, T. E. Cowan, K. G. Estabrook, B. A. Hammel, S. P. Hatchett, E. A. Henry, D. E. Hinkel, J. D. Kilkenny, J. A. Koc, W. L. Kruer, A. B. Langdon, B. F. Lasinski, R. W. Lee, M. B. J., A. MacKinnon, J. D. Moody, M. J. Moran, A. A. Offenbergr, D. M. Pennington, M. D. Perry, T. J. Phillips, T. C. Sangster, M. S. Singh, M. A. Stoyer, M. Tabak, G. L. Tietbohl, M. Tsukamoto, K. Wharton, and S. C. Wilks, "Hot electron production and heating by hot electrons in fast ignitor research," Physics of Plasmas 5, 1966 (1998).

${ }^{17}$ G. Cristoforetti, M. P. Anania, A. Y. Faenov, A. Giulietti, D. Giulietti, S. B. Hansen, P. Koester, L. Labate, T. Levato, T. A. Pikuz, and L. A. Gizzi, "Spatially resolved analysis of $\mathrm{K}$ alpha $\mathrm{x}$-ray emission from plasmas induced by a femtosecond weakly relativistic laser pulse at various polarizations," Physical Review E 87 (2013), 10.1103/PhysRevE.87.023103.

${ }^{18}$ P. Köster, K. Akli, D. Batani, S. Baton, R. G. Evans, A. Giulietti, D. Giulietti, L. A. Gizzi, J. S. Green, M. Koenig, L. Labate, A. Morace, P. Norreys, F. Perez, J. Waugh, N. Woolsey, and K. L. Lancaster, "Experimental investigation of fast electron transport through $\mathrm{K} \alpha$ imaging and spectroscopy in relativistic laser-solid interactions," Plasma Physics and Controlled Fusion 51 (2009), 10.1088/0741-3335/51/1/014007.

${ }^{19}$ K. B. Wharton, S. P. Hatchett, S. C. Wilks, M. H. Key, J. D. Moody, V. Yanovsky, A. A. Offenberger, B. A. Hammel, M. D. Perry, and C. Joshi, "Experimental measurements of hot electrons generated by ultraintense $\left(>10^{19} \mathrm{~W} / \mathrm{cm}^{2}\right)$ laser-plasma interractions on solid-density targets," Physical Review Letters 81, 822 (1998).

${ }^{20}$ S. Baton, A. Colaitis, C. Rousseaux, G. Boutoux, S. Brygoo, L. Jacquet, M. Koenig, D. Batani, A. Casner, E. Le Bel, D. Raffestin, V. Tikhonchuk, J. Trela, C. Reverdin, L. LeDeroff, W. Theobald, G. Cristoforetti, L. A. Gizzi, P. Koester, L. Labate, and K. Shigemori, "Preliminary results from the LMJ-PETAL experiment on hot electrons characterization in the context of shock ignition," High Energy Density Physics 36, 100796 (2020). 
${ }^{21}$ J. Trela, W. Theobald, K. S. Anderson, D. Batani, R. Betti, A. Casner, J. A. Delettrez, J. A. Frenje, Y. Glebov, X. Ribeyre, A. A. Solodov, M. Stoeckl, and C. Stoeckl, "The control of hot-electron preheat in shock-ignition implosions," Physics of Plasmas 25, 052707 (2018).

${ }^{22}$ C. D. Chen, J. A. King, M. H. Key, K. U. Akli, F. N. Beg, H. Chen, R. R. Freeman, A. Link, A. J. Mackinnon, A. G. MacPhee, P. K. Patel, M. Porkolab, R. B. Stephens, and L. D. Van Woerkom, "A bremsstrahlung spectrometer using K-edge and differential filters with image plate dosimeters," Review of Scientific Instruments 79, 10E305 (2008).

${ }^{23}$ C. Courtois, R. Edwards, C. Compant La Fontaine, C. Aedy, S. Bazzoli, J. L. Bourgade, J. Gazave, J. M. Lagrange, O. Landoas, L. Le Dain, D. Mastrosimone, N. Pichoff, G. Pien, and C. Stoeckl, "Characterisation of a MeV bremsstrahlung x-ray source produced from a high intensity laser for high areal density object radiography," Physics of Plasmas 20, 083114 (2013).

${ }^{24}$ R. H. H. Scott, E. L. Clark, F. Pérez, M. J. V. Streeter, J. R. Davies, H.-P. Schlenvoigt, J. J. Santos, S. Hulin, K. L. Lancaster, S. D. Baton, S. J. Rose, and P. A. Norreys, "Measuring fast electron spectra and laser absorption in relativistic laser-solid interactions using differential bremsstrahlung photon detectors," Review of Scientific Instruments 84, 083505 (2013).

${ }^{25}$ Y. J. Rhee, S. M. Nam, J. Peebles, H. Sawada, H. Wei, X. Vaisseau, T. Sasaki, L. Giuffrida, S. Hulin, B. Vauzour, J. J. Santos, D. Batani, H. S. McLean, P. K. Patel, Y. T. Li, D. W. Yuan, K. Zhang, J. Y. Zhong, C. B. Fu, N. Nua, K. Li, Y. Zhang, J. Q. Zhu, I. J. Kim, J. H. Jeon, T. M. Jeong, I. W. Choi, H. W. Lee, J. H. Sung, S. K. Lee, and C. H. Nam, "Spectral tomographic analysis of bremsstrahlung X-rays generated in a laser produced plasma," Laser and Particle Beams 34, 645-654 (2016).

${ }^{26}$ O. Klimo, J. Psikal, V. T. Tikhonchuk, and S. Weber, "Two-dimensional simulations of laser-plasma interaction and hot electron generation in the context of shock-ignition research," Plasma Physics and Controlled Fusion 56, 055010 (2014).

${ }^{27}$ S. Agostinelli, J. Allison, K. Amako, J. Apostolakis, H. Araujo, P. Arce, M. Asai, D. Axen, S. Banerjee, G. Barrand, F. Behner, L. Bellagamba, J. Boudreau, L. Broglia, A. Brunengo, H. Burkhardt, S. Chauvie, J. Chuma, R. Chytracek, G. Cooperman, G. Cosmo, P. Degtyarenko, A. Dell'Acqua, G. Depaola, D. Dietrich, R. Enami, A. Feliciello, C. Ferguson, H. Fesefeldt, G. Folger, F. Foppiano, A. Forti, S. Garelli, S. Giani, R. Giannitrapani, 
D. Gibin, J. J. Gomez Cadenas, I. Gonzalez, G. Gracia Abril, G. Greeniaus, W. Greiner, V. Grichine, A. Grossheim, S. Guatelli, P. Gumplinger, R. Hamatsu, K. Hashimoto, H. Hasui, A. Heikkinen, A. Howard, V. Ivanchenko, A. Johnson, F. W. Jones, J. Kallenbach, N. Kanaya, M. Kawabata, Y. Kawabata, M. Kawaguti, S. Kelner, P. Kent, A. Kimura, T. Kodama, R. Kokoulin, M. Kossov, H. Kurashige, E. Lamanna, T. Lampen, V. Lara, V. Lefebure, F. Lei, M. Liendl, W. Lockman, F. Longo, S. Magni, M. Maire, E. Medernach, K. Minamimoto, P. Mora de Freitas, Y. Morita, K. Murakami, M. Nagamatu, R. Nartallo, P. P. Nieminen, T. Nishimura, K. Ohtsubo, M. M. Okamura, S. O’Neale, Y. Oohata, K. Paech, J. Perl, A. Pfeiffer, M. G. Pia, F. Ranjard, A. Rybin, S. Sadilov, E. Di Salvo, G. Santin, T. Sasaki, N. Savvas, Y. Sawada, S. Scherer, S. Sei, V. Sirotenko, D. Smith, N. Starkov, H. Stoecker, J. Sulkimo, M. Takahata, S. Tanaka, E. Tcherniaev, E. Safai Tehrani, M. Tropeano, P. Truscott, H. Uno, L. Urban, P. Urban, M. Verderi, A. Walkden, W. Wander, H. Weber, J. P. Wellisch, T. Wenaus, D. C. Williams, D. Wright, T. Yamada, H. Yoshida, and D. Zschiesche, "GEANT4 - a simulation toolkit," Nuclear Instruments and Methods in Physics Research A 506, 250-303 (2003).

${ }^{28}$ F. Salvat, J. M. Fernandez-Varea, and J. Sempau, PENELOPE-2011: A Code System for Monte Carlo Simulation of Electron and Photon Transport, OECD NEA Data Bank/NSC DOC(2011)/5 (Organisation for Economic Co-Operation and Development - Nuclear Energy Agency, Issy-les-Moulineaux, France, 2011).

${ }^{29}$ L. Pandola, C. Andenna, and B. Caccia, "Validation of the GEANT4 simulation of bremsstrahlung from thick targets below $3 \mathrm{MeV}$," Nuclear Instruments and Methods in Physics Research B 350, 41-48 (2015).

${ }^{30}$ G. J. Williams, B. R. Maddox, H. Chen, S. Kojima, and M. Millecchia, "Calibration and equivalency analysis of image plate scanners," Review of Scientific Instruments 85, 11E604 (2014).

${ }^{31}$ B. Hidding, G. Pretzler, M. Clever, F. Brandl, F. Zamponi, A. Lubcke, T. Kampfer, I. Uschmann, E. Forster, U. Schramm, R. Sauerbrey, E. Kroupp, L. Veisz, K. Schmid, S. Benavides, and S. Karsch, "Novel method for characterizing relativistic electron beams in a harsh laser-plasma environment," Review of Scientific Instruments 78, 083301 (2007). ${ }^{32}$ G. Boutoux, N. Rabhi, D. Batani, A. Binet, J.-E. Ducret, K. Jakubowska, J.-P. Négre, C. Reverdin, and I. Thfoin, "Study of imaging plate detector sensitivity to 5-18 MeV electrons," Review of Scientific Instruments 86, 113304 (2015). 
${ }^{33}$ G. Boutoux, D. Batani, F. Burgy, J.-E. Ducret, P. Forestier-Colleoni, S. Hulin, N. Rabhi, A. Duval, L. Lecherbourg, C. Reverdin, K. Jakubowska, C. I. Szabo, S. Bastiani-Ceccotti, F. Consoli, A. Curcio, R. De Angelis, F. Ingenito, J. Baggio, and D. Raffestin, "Validation of modelled imaging plates sensitivity to 1-100 keV x-rays and spatial resolution characterisation for diagnostics for the "PETawatt Aquitaine Laser"," Review of Scientific Instruments 87, 043108 (2016). 


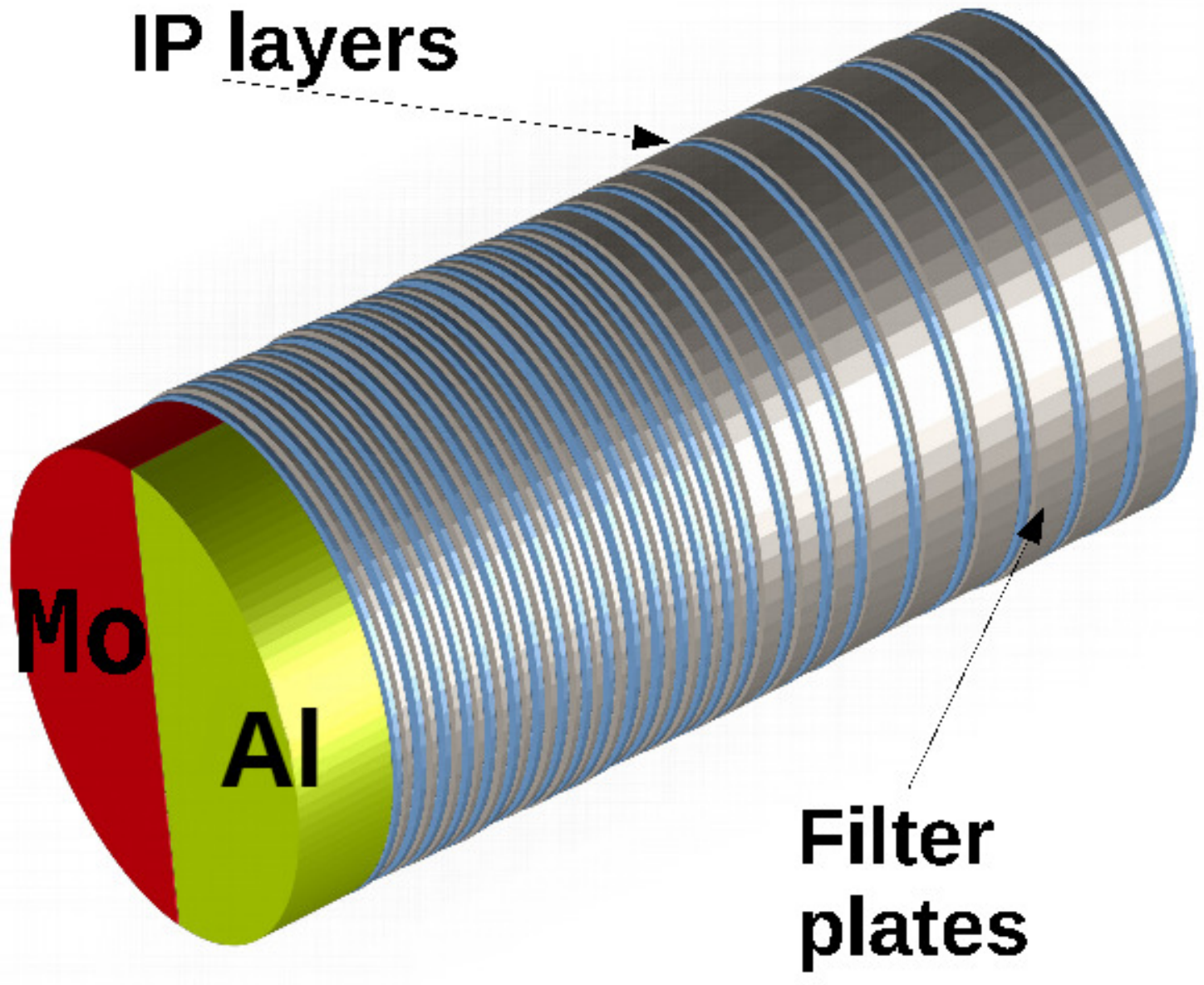




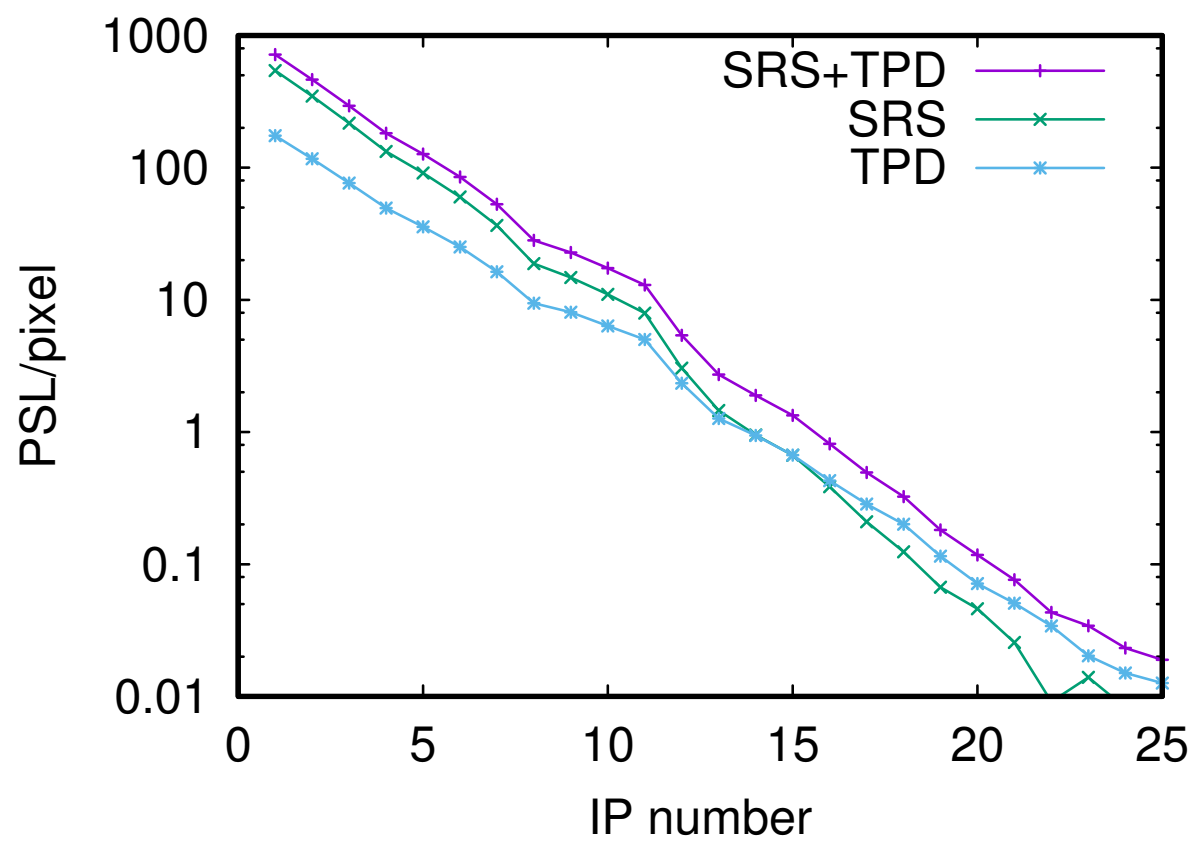




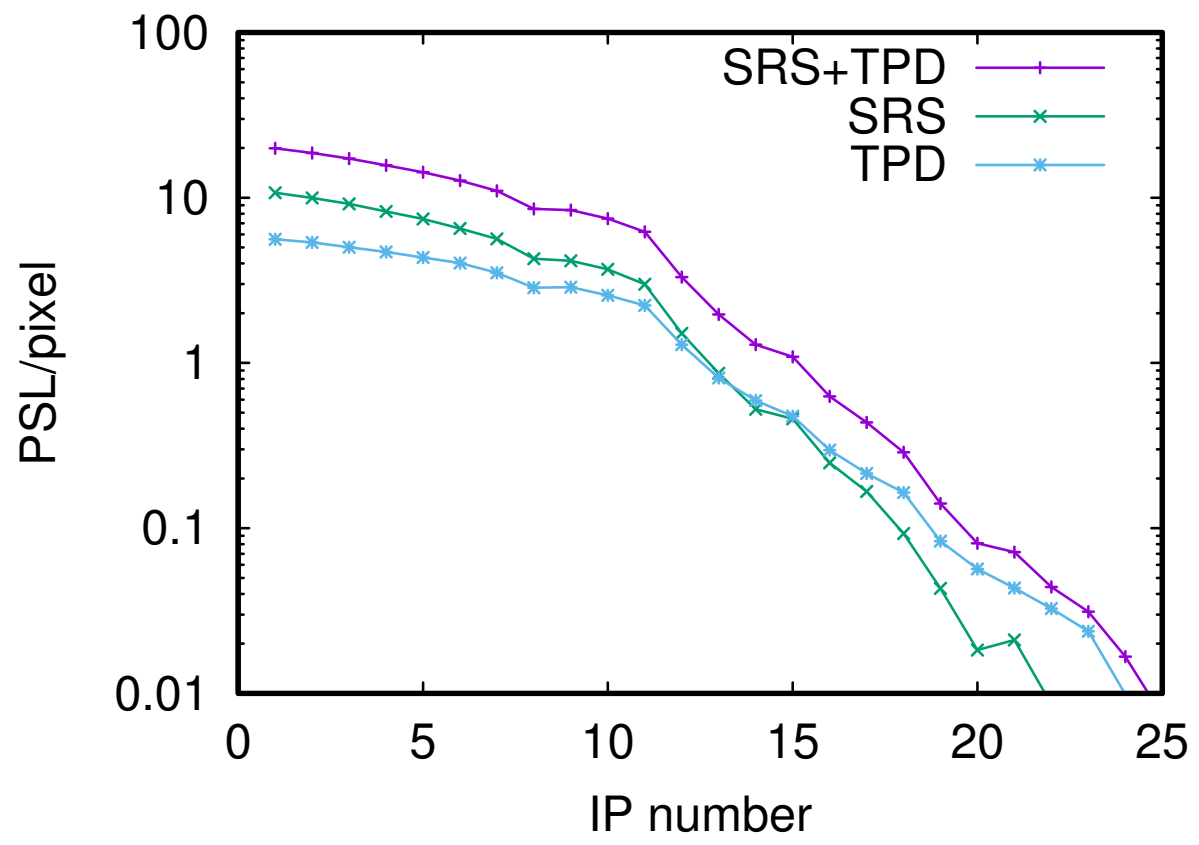




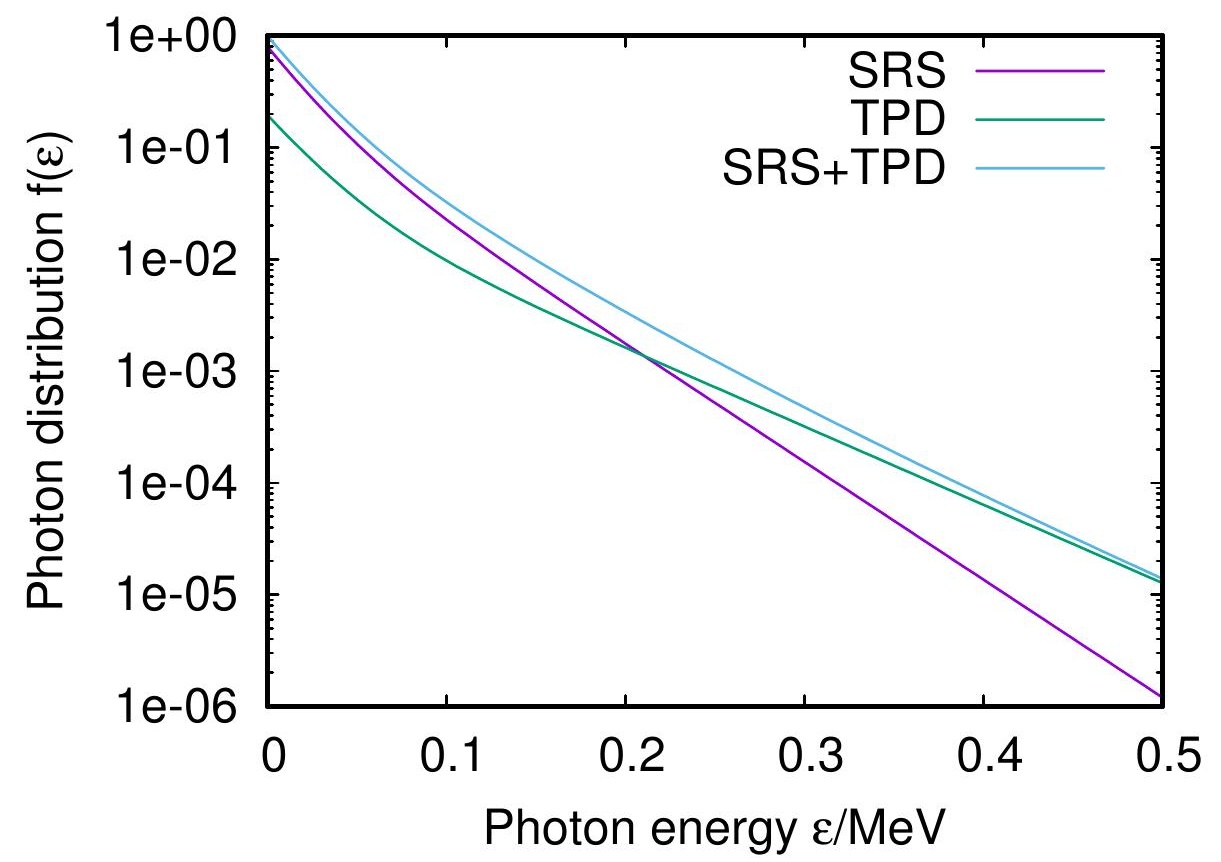




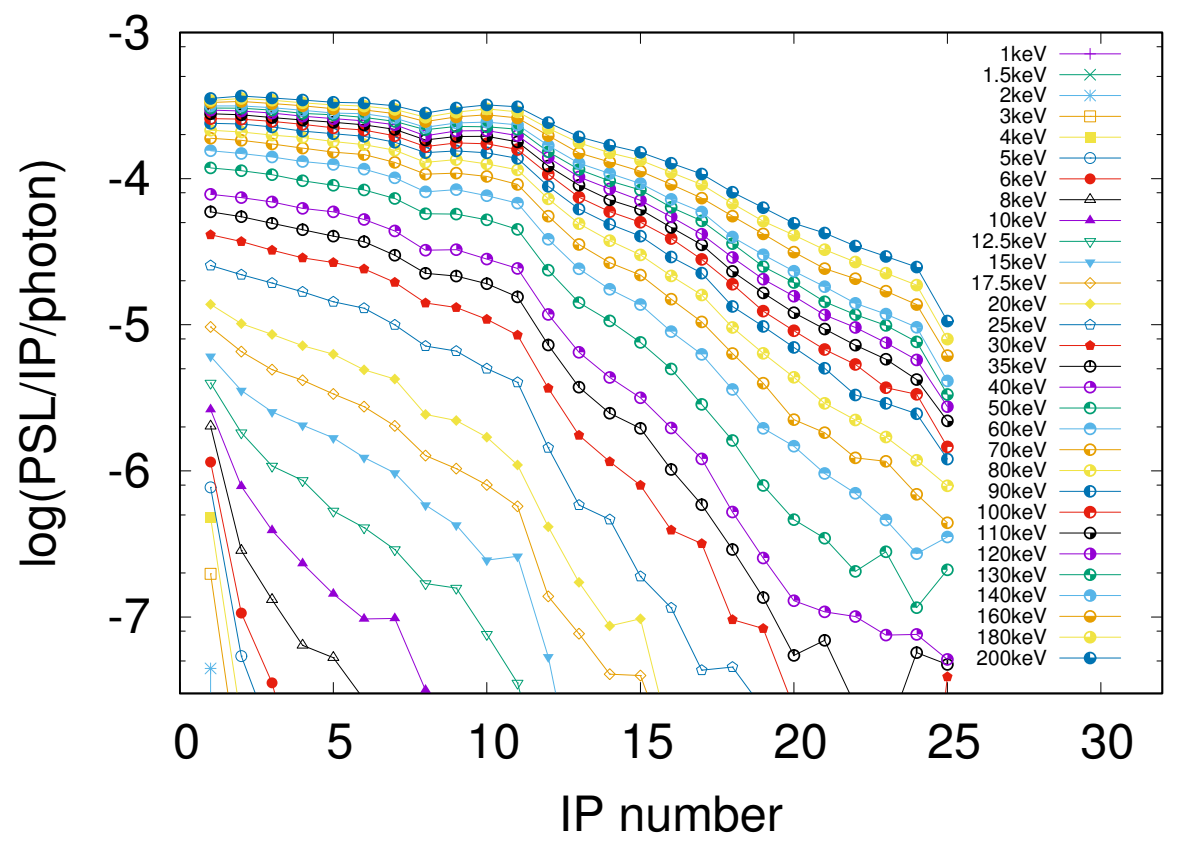



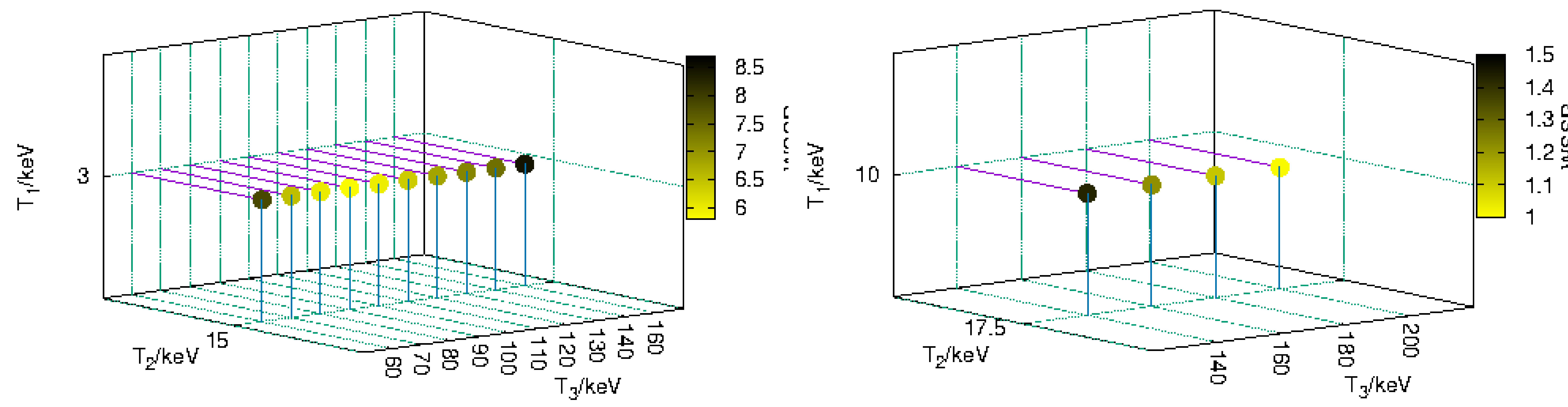

Shot2: Al channel ( $T_{1}=3 \mathrm{kev}$ )

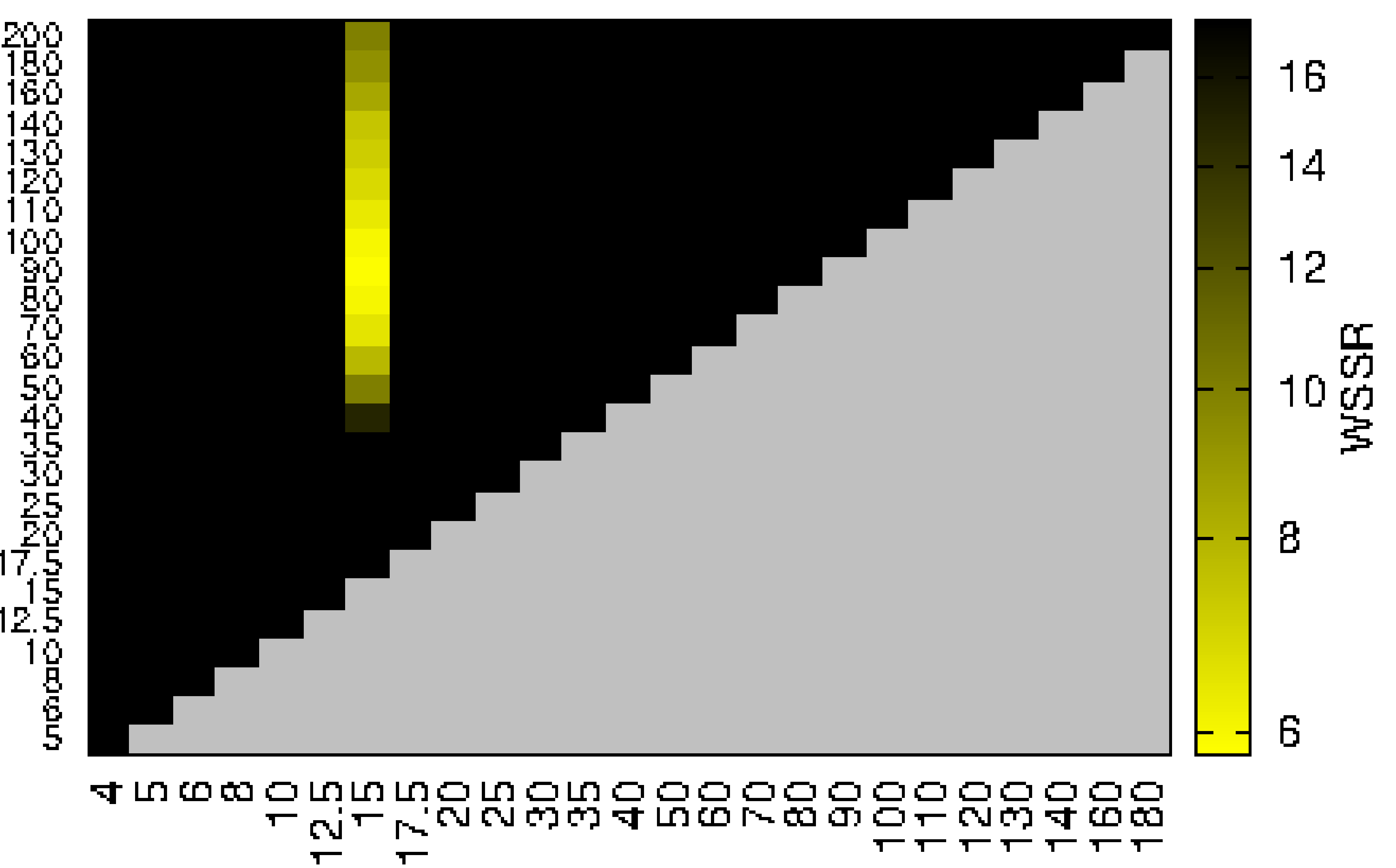

$\mathrm{T}_{2} \mathrm{keV}$
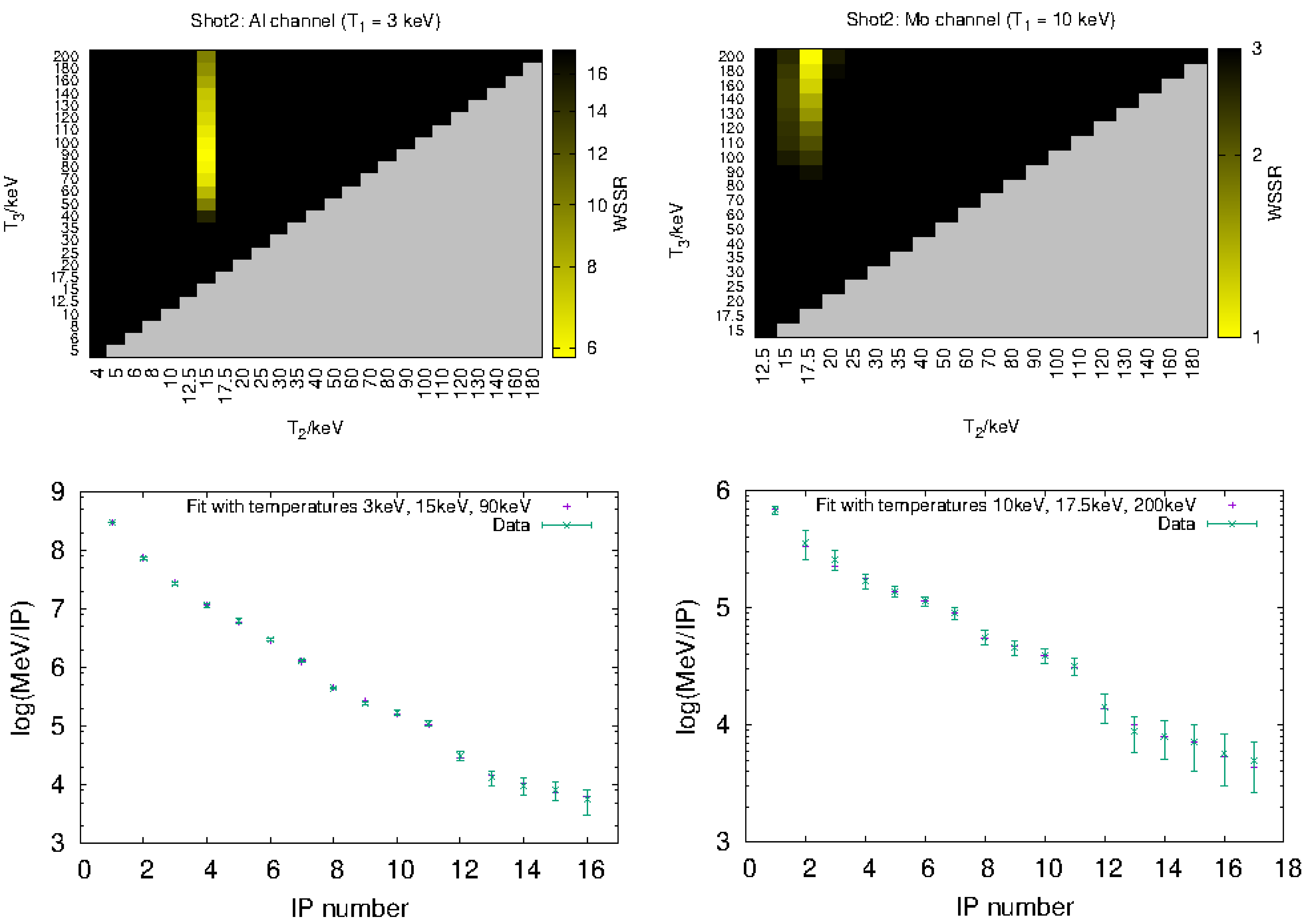

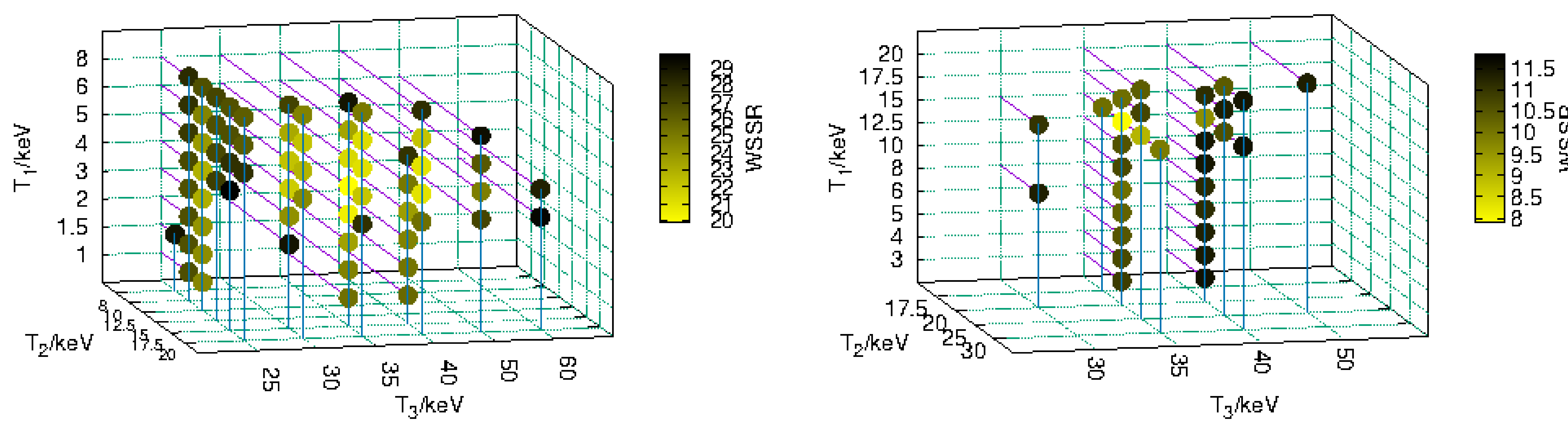

Shot3: Al channel ( $\mathrm{T}_{1}=4 \mathrm{kev}$ )

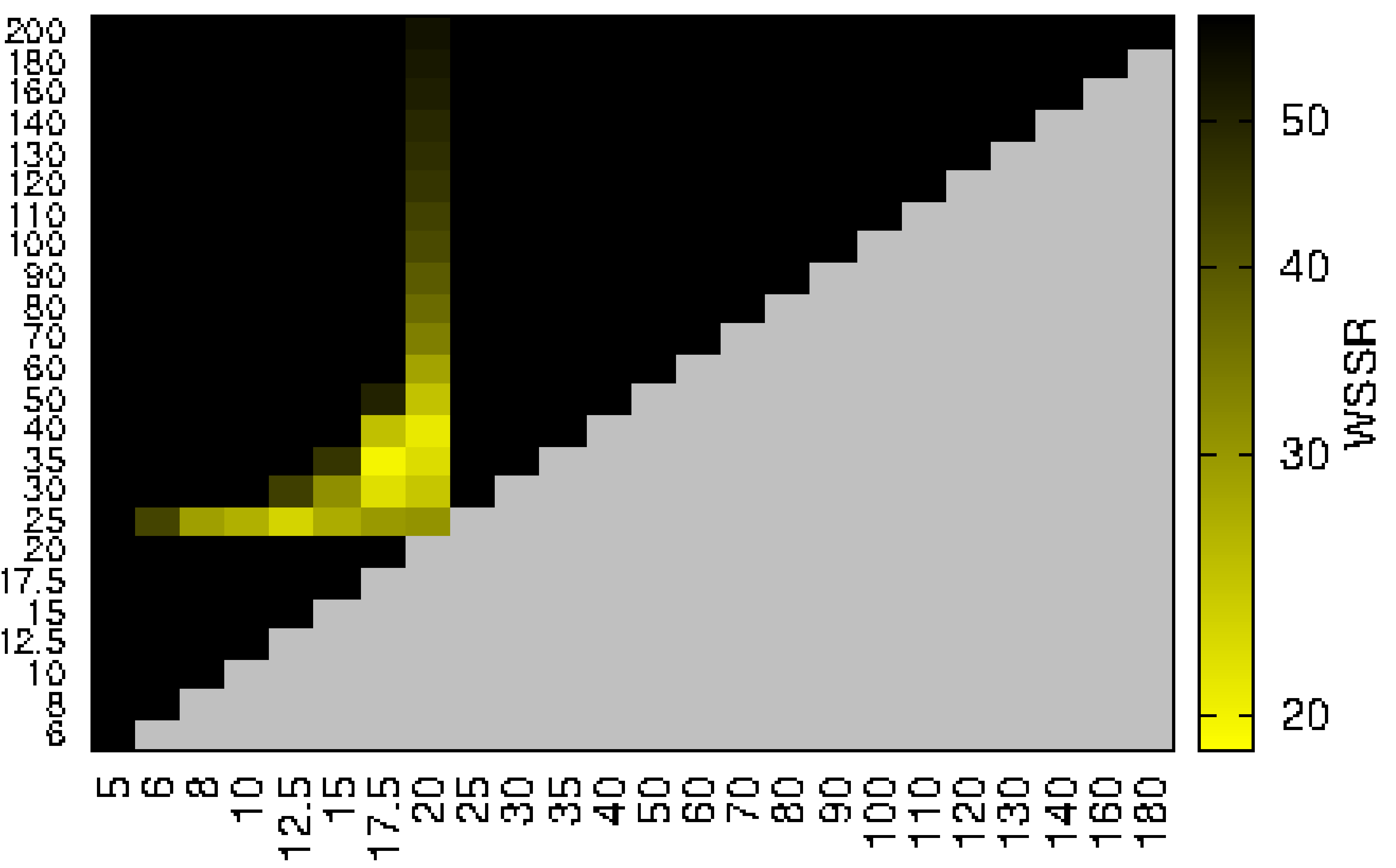

$T_{2}$ kev
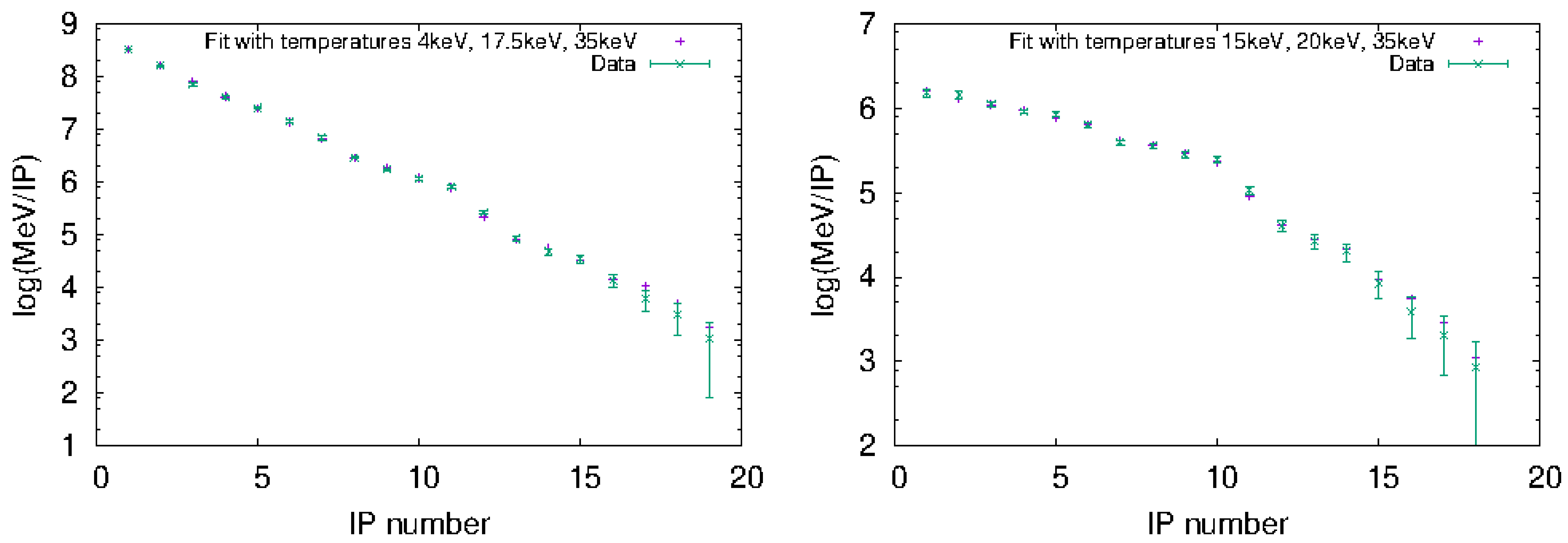
